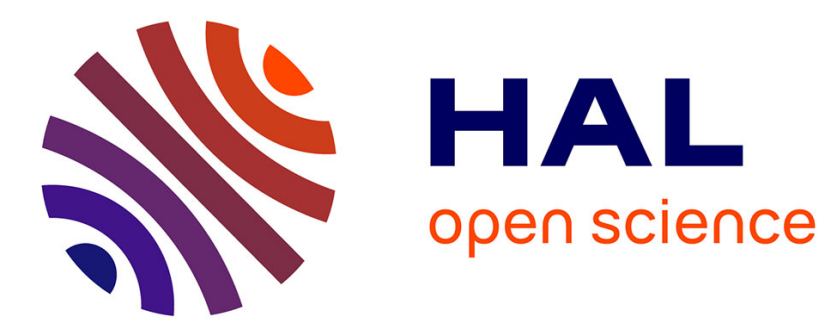

\title{
Essai sur le développement topographique de la nécropole protohistorique de Pézenas (Hérault)
}

André Nickels

\section{To cite this version:}

André Nickels. Essai sur le développement topographique de la nécropole protohistorique de Pézenas (Hérault). Gallia - Fouilles et monuments archéologiques en France métropolitaine, 1990, 47, pp.1-27. 10.3406/galia.1990.2900 . hal-01916256

\section{HAL Id: hal-01916256 \\ https://hal.science/hal-01916256}

Submitted on 20 Jan 2020

HAL is a multi-disciplinary open access archive for the deposit and dissemination of scientific research documents, whether they are published or not. The documents may come from teaching and research institutions in France or abroad, or from public or private research centers.
L'archive ouverte pluridisciplinaire HAL, est destinée au dépôt et à la diffusion de documents scientifiques de niveau recherche, publiés ou non, émanant des établissements d'enseignement et de recherche français ou étrangers, des laboratoires publics ou privés.

\section{(1) (1) $\$$}

Distributed under a Creative Commons Attribution - NonCommercial - NoDerivatives| 4.0 


\title{
ESSAI SUR LE DÉVELOPPEMENT TOPOGRAPHIQUE DE LA NÉCROPOLE PROTOHISTORIQUE DE PÉZENAS (HÉRAULT)
}

\author{
par André NICKELS †
}

La nécropole protohistorique de Pézenas, explorée de 1963 à 1970, n'a fait l'objet, jusqu'à ce jour, que de travaux très ponctuels, souvent limitès à l'étude de telle ou telle catégorie de mobilier, sans que cet exceptionnel gisement soit pris en compte dans sa globalité. Une étude détaillée de la répartition des diverses catégories de mobilier, céramiques et objets métalliques, permet pourtant de bien mettre en évidence quatre grandes phases dans le développement topographique de cette nécropole. Grâce aux céramiques grecques et étrusques qui figurent en abondance dans les tombes, ces différentes étapes peuvent être parfaitement datées entre la fin du vir" et les débuts du v's. L'étude globale des ensembles funéraires, phase par phase, permet ainsi, non seulement de bien dater les changements dans les rites funéraires qui marquent le Premier Age du Fer, mais jette également une lumière nouvelle sur les modifications que produit, au sein même des populations locales, la multiplication des contacts et des échanges avec les navigateurs méditerranéens, Grecs ou Étrusques.

The protohistoric necropolis of Pezenas, explored from 1963 to 1970, has so far only given rise to a few partial publications about one category of furniture or other without any comprehensive study of this outstanding site. A close scruting of the various tomb contents, earthenware and metallic furniture, highlights four stages in the topographic development of the necropolis. Thanks to the Greek and Etruscan earthenware which is widely represented in the lombs, these four stages can be accuretely dated, ranging from the late 7th to the early 5th century. The exhaustive study of the funeral sets, stage after stage, enables us to date the changes in the funeral rites during the early Iron Age and throws a new light on the transformation within the local population resulting from the increasing contacts with Mediterranean sailors whether Greek or Etruscan.

La célèbre nécropole protohistorique de Pézenas (fig. 1) a èté découverte fortuitement en octobre 1963, à l'occasion de travaux agricoles, dans une parcelle du lieu-dit «Saint-Julien» (fig. 2) ${ }^{1}$. Son exploration a été menée, pour l'essentiel, par J. Giry de 1963 à 1965 et complétée en 1969 par une nouvelle équipe sous la direction de Chr. Llinas. Au

1 Pour la localisation précise de la nécropole, voir Chr. Lisnas et. A. Rorert, La nécropole de Saint-Julien à Pézenas (Hérault). Fouilles de 1969 et 1970, Revue Archéologique de Narbonnaise, 1971, p. 1-33 et fig. 2. total, elle a livré plus de 200 tombes qui couvrent tout le $\mathrm{vi}^{\mathrm{e}}$ et les débuts du ve s. (fig. 3). Dès 1965, J. Giry publiait un inventaire très complet de tout le mobilier découvert dans les sépultures ${ }^{2}$. Cet inventaire, s'il n'est pas illustré, est cependant très

2 J. GirY, La nécropole pré-romaine de Saint-Julien (commune de Pézenas, Hérault), Revue des Éludes Ligures, XXXI, 1965, p. 117-238. Voir également du même auteur, La tombe 11 de la nécropole "Saint-Julien" à Pézenas, $X X X I X^{e}$ Congrès de la Fédération Historique du Languedoc Méditerranéen et du Roussillon, 1967, p. 59-64. 


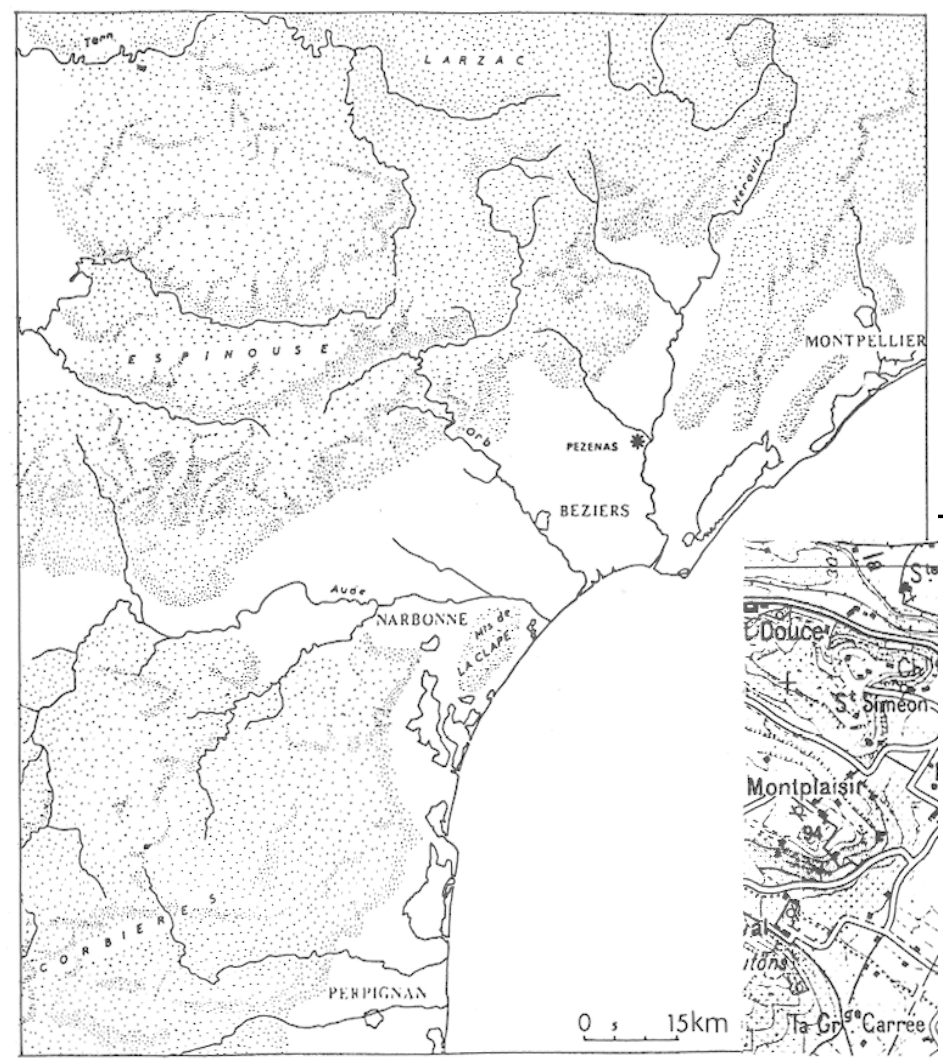

Fig. 1 - Situation géographique du gisement protohistorique de Pézenas.

Fig. 2 - Extrait de la carte I.G.N. au 1/50000" avec la localisation de la nécropole.

\section{Illustration non autorisée à la diffusion}

précieux : les vases et objets métalliques y sont décrits avec soin et leurs dimensions méthodiquement relevées. Le mobilier découvert aux abords des tombes y est, en outre, décompté et analysé avec la même précision. Chr. Llinas et A. Robert ont, quant à eux, livré, dès 1971, un compte rendu détaillé des dernières campagnes de fouilles ${ }^{3}$. Nous disposons, de plus, de très nombreuses descriptions de séries de mobilier, souvent extrêmement détaillées - mais peu illustrées - livrées par tel ou tel chercheur au gré de ses intérêts du moment et qui fournissent une documentation globablement abondante - sinon complète - sur cet exceptionnel ensemble funéraire protohistorique ${ }^{4}$. Une récente recherche d'ensemble,

3 Chr. Llinas et A. Robert, op. cit.

4 On consultera ainsi, pour le bucchero nero : A. RoBert, La place du bucchero nero dans la nécropole de Saint-Julien de Pézenas (Hérault), in: Le bucchero nero étrusque et sa diffusion en Gaule Méridionale, coll. Latomus, vol. 160, Bruxelles, 1979, p. 139-146; -- pour les gobelets carénés gris, J.-J. Jully et Y. SolieR, Les gobelets gris carénés, faits au tour, à l'Age du Fer languedocien, Revue des Etudes Ligures, XXXIII, 1-3, p. 217-244; - pour les céramiques non tournées, B. DEDET, La céramique non tournée de la nécropole de Saint-Julien à Pézenas (Héraull) dans son contexte languedocien au $v^{e} s$. av. J.-C., thèse de $3^{e}$ cycle, Univ. de Montpellier, 1974; - pour la céramique tournée, l'ouvrage de J.-J. Jur.y, Céramiques grecques ou de type grec et autres menée sur le développement topographique des cimetières du Premier Age du Fer, dans le cadre de la préparation d'une publication ponctuelle ${ }^{5}, \mathrm{~m}$ 'a conduit à rassembler et à exploiter cette immense documentation éparse. Les résultats obtenus pour Pézend́s dépassent très largement le cadre de cette seule nécropole et intéressent l'ensemble des recherches menées sur l'occupation protohistorique du Languedoc méditerranéen.

Les différents travaux consacrés jusqu'à présent à la nécropole de Pézenas ont systématiquement limité leur ambition à dater les diverses catégories de mobilier étudiées par le jeu de l'association au sein d'une même tombe avec du mobilier présumé mieux

céramiques en Languedoc méditerranéen, Roussillon et Calalogne, $V_{I I} I_{-I} V^{e}$ s., Besançon, 1983, p. 697-778 et 1305-1320, fournit un inventaire détaillé exhaustif, malheureusement peu illustré, qui reprend toutes les publications ponctuelles consacrées par cet auteur à la nécropole de Pézenas, notamment J.-J. Juı..Y, Vases stamnoïdes de type grec archaïque en provenance d'une nécropole languedocienne de la fin du I"r Age du Fer, Monuments Piot, 61, 1977, p. 1-24 et J.-J. Jur.I.Y, Vases grecs en provenance de sépultures pré-romaines en Languedoc et en Catalogne (fin vire-rve s. av. J.-C.), L'Antiquité Classique, 46/1, 1977 , p. 5-40.

5 A. Nickeis, G. Marchand et M. Schwal.l.er, Agde, la nécropole du premier Age du Fer, Paris, 1989. 


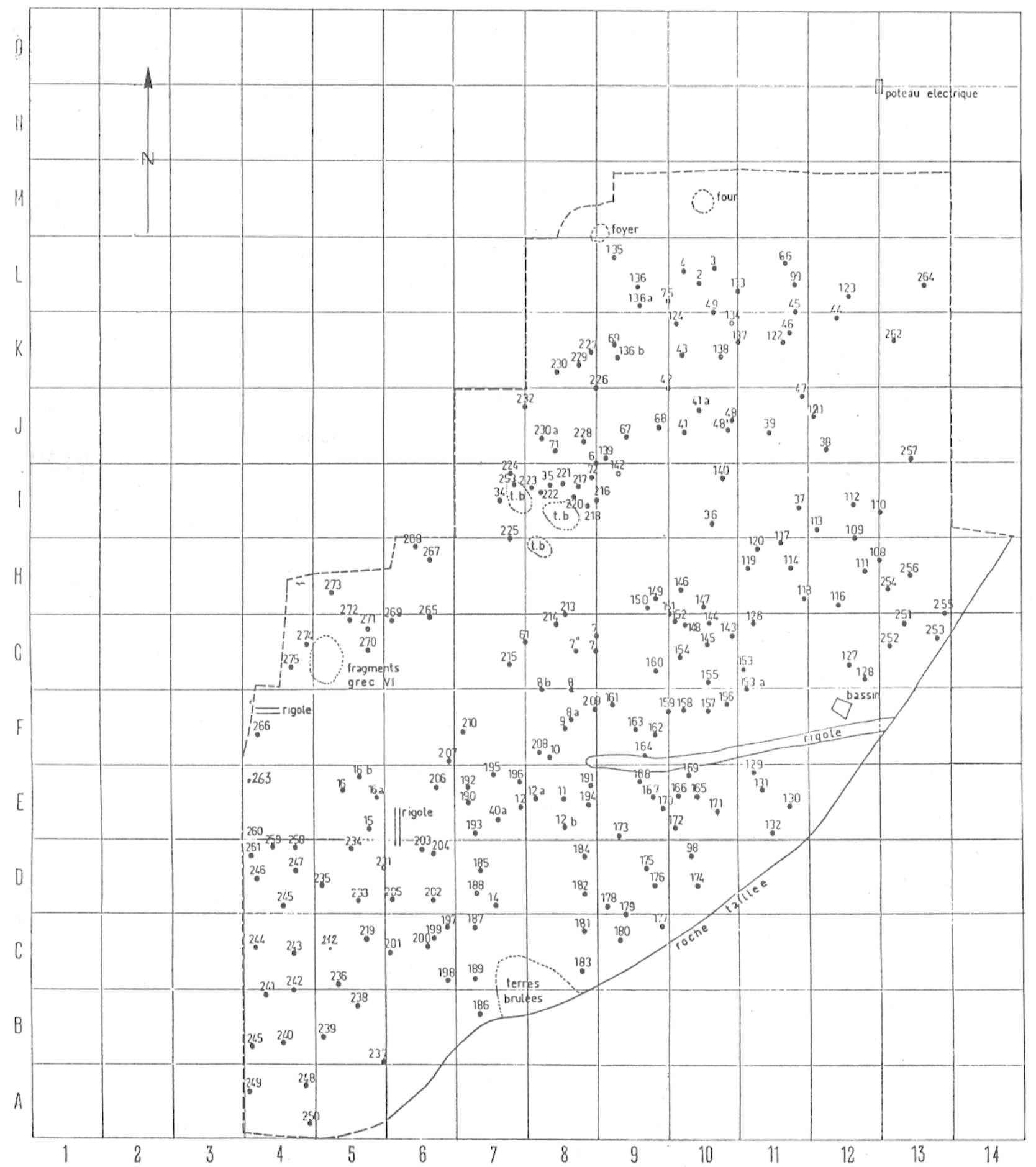

Fig. 3 - Plan général de la nécropole de Pézenas d'après J. Giry.

daté : céramiques par rapport aux objets métalliques, céramiques modelées par rapport aux céramiques tournées, céramiques grecques d'Occident par rapport aux séries importées. Lorsqu'une mise en phase a été tentée, elle l'a toujours été de façon strictement interne, en lonction du seul mobilier des tombes ${ }^{6}$. Aucune de ces études ne s'est attachée à comprendre la dynamique de développement propre à cet ensemble funéraire. Comme on le sait, à la

6 Voir, à ce sujet, J.-J. Jully, Céramiques grecques ou de type grec..., op. cil., p. 1316-1320. 
différence des habitats qui, en règle générale, se développent par superposition progressive des divers niveaux d'occupation, les nécropoles présentent normalement un développement horizontal, nucléaire dans le cas de groupes familiaux, global lorsque le cimetière s'étend par simple ajout de tombes à la périphérie du secteur occupé précédemment? Comprendre la logique de tel ou tel type de développement présente évidemment le plus grand intérêt.

Pour ma part, j'ai donc choisi, dans un premier temps, de mettre en évidence la chronologie relative des diverses zones d'ensevelissement. Dans un second temps, j'essaierai d'ancrer cette chronologie relative dans une chronologie absolue, toujours susceptible, quant à elle, d'être complétée ou précisée au fur et à mesure de l'avancement des recherches.

\section{MÉTHODE D'INVESTIGATION ET CRITERESS D'ANALYSE}

Pour aborder le problème du développement topographique de ce gisement, j'ai eu recours à des "collections de cartes" sur le modèle utilisé par les géographes, notamment en géographie rurale. Le premier travail consiste à définir un certain nombre de critères discriminants rigoureux. Il peut s'agir de particularités d'aménagement, ou encore de catégories de mobilier. Chaque critère retenu fera l'objet d'une carte de répartition, qui traduira la dispersion spatiale de cet élément à l'intérieur de la nécropole. La comparaison des diverses cartes, par simple juxtaposition, ou mieux, par superposition, permettra de déceler toutes sortes de groupements, de concentrations spatiales - ou au contraire de dispersions - qu'il conviendra d'analyser avec le plus grand soin. Bien entendu, ces cartes seront d'autant plus précises que les catégories de mobilier auront èté définies avec soin. L'interprétation de ces documents devra, évidemment, rester prudente. La concentration d'un certain type d'objet dans un secteur limité peut, en effet, avoir des significations très diverses, voire contradictoires. Il conviendra, à chaque fois, de vérifier que l'anomalie est bien de nature chronologique et non, par exemple, de nature ethnologique ou sexuelle.

7 Sur ces questions. je renvoie, pour mémoire, aux travaux de H. Müller-Karpe, notamment, Beilräge zur Chronologie der Urnenfelderzeil nördlich und südlich der Alpen, Berlin, 1959.
Les figures $\mathrm{n}^{\text {os }} 4$ à 11 présentent les cartes établies à partir des renseignements dont je disposais pour la nécropole de Pézenas ${ }^{8}$. Je complèterai chacun de ces documents par un bref commentaire définissant le critère retenu et la signification des particularités de répartition observées. La confrontation générale de tous ces documents permettra ensuite de dégager les grandes lignes de l'évolution topographique du gisement.

\section{LES CÉRAMIQUES MODELÉES}

Cette catégorie de mobilier est bien connue grâce au travail de détail qui lui a été consacré par B. Dedet ${ }^{9}$. Le matériel céramique y est entièrement. dessiné et les associations sont indiquées avec précision. Les formes de vases ou les éléments décoratifs ou morphologiques dont la répartition a été examinée sont les suivants ${ }^{10}$.

La décoration de cannelures fines (fig. $4 \mathrm{~b}$ ). Tous les décors de ce type seront pris en comple quel que soil leur emplacement sur le vase. La carte de répartition de cette particularité décorative indique une très remarquable concentration dans la partie sud de la nécropole. Aucune sépulture des zones nord-est, nord et nord-ouest ne contient de vase présentant ce type d'aménagement.

Les urnes à pied (fig. 4a et 4c). De façon générale, on constate que les urnes à pied se concentrent dans la partie sud de la nécropole. Cette forme est, en revanche, totalement absente des autres secteurs. Dans le détail, on peut distinguer les véritables cratères à pied haut des urnes à pied bas, dont la répartition est légèrement différente. Ces dernières (fig. 4c) n'apparaissent en effet qu'à la périphérie du secteur de concentration maximale des cratères à pied haut (fig. 4a).

Les coupelles à fond ombiliqué ou à fond plat. La carte de dispersion des diverses variantes de ce type de récipient montre une répartition égale des coupelles à fond ombiliqué et à fond plat. L'orientation du

8 Je me suis attaché, en particulier, à cartographier tout le mobilier découvert aux abords des tombes qui a été très méticuleusement décompté par J. Giry. Grâce à ce travail, long et souvent fastidieux, le matériel dispersé par les labours anciens ou modernes a pu être assez facilement éliminé. Cette partie de la documentation ne sera pas présentée ici afin de ne pas alourdir inutilement notre étude.

9 B. DEDET, La céramique non tournée de la nécropole de Saint-Julien..., op. cit.

10 Nous ne fournirons ici que les cartes qui ont donné des résultats positifs. Les répartitions non significatives seront simplement signalées mais non illustrées. 

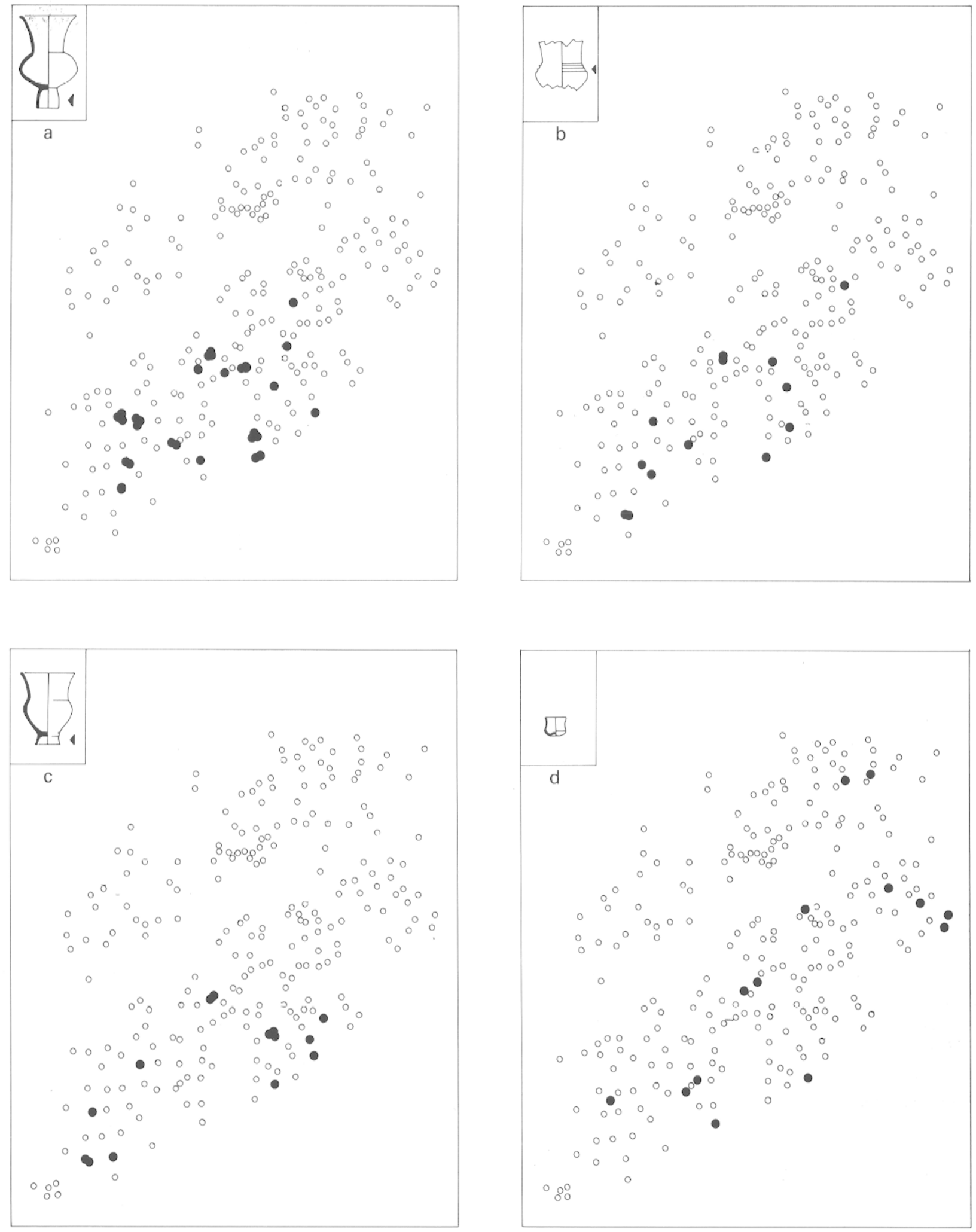

Fig. 4 - Cartes de répartition des urnes à pied haut (a), des décors de cannelures (b), des urnes à pied bas (c) et des gobelets carénés (d). 
bord, divergent, droit ou convergent ne paraît pas non plus avoir de signification chronologique, du moins apparente, les divers types apparaissant avec la même régularité dans tout le secteur de diffusion des coupelles : il s'agit donc là d'un critère non discriminant. On remarquera, en outre, que ces coupelles se trouvent dans une zone assez vaste qui englobe la partie nord de la nécropole où les cratères à pieds et le décor de cannelures sont absents.

Les urnes à décor d'indentations sur le haut de la panse. On distinguera ici deux types de décors, les incisions fines, longues, et les indentations larges et courtes. Si ces dernières sont répandues dans une très grande partie de la nécropole, les premières ne sont attestées que dans la partie sud, dans un secteur très limité.

Les coupes à pied haut. I.eur diffusion touche essentiellement les parties sud et nord-est du gisement. Elle coïncide avec celle des coupelles mais est plus vaste que celle des cratères à pied haut et du décor de cannelures.

Les gobelets. D'un point de vue morphologique, on distingue diverses variantes dont trois seulement sont assez bien attestées pour être prises en considération ici :

- gobelets carénés (fig. 4d). Ils possèdent une paroi concave et une carène basse bien marquée, immédiatement au-dessus de la base plane. Leur zone de répartition dans la nécropole est beaucoup plus vaste que celle des types précédents. Dans la partie sud, ils n'apparaissent qu'à la périphérie de la zone occupée par les types précédents, mais sont également présents dans les secteurs nord-est et nord. Ils sont, en revanche, totalement absents de la zone centrale et de la partie nord-ouest;

- gobelets tronconiques (fig. 5a). Ils ont des parois légèrement obliques et un fond plat. Ce type de récipient n'apparaît que dans la partie la plus méridionale de la nécropole, dans une aire très limitée ;

- gobelets ovoïdes à lèvre évasée (fig. 5a). Ces récipients de forme ovoïde possèdent un fond plat et une lèvre légèrement évasée. Ils se concentrent à nouveau tous dans la partie sud de la nécropole, dans une aire très bien définie, qui est cependant différente de celle des gobelets tronconiques à fond plat. Il s'agit donc là encore d'un critère discriminant intéressant.

Les tombes à mobilier exclusivement indigène (fig. 5b). La carte de répartition est très significative : les tombes qui ne livrent que des vases modelés sont toutes concentrées dans les secteurs sud et nord-est de la nécropole.

L'examen de cette première série de documents apporte déjà quelques éléments de réflexion. La superposition de ces cartes permet, en effet, d'opposer deux zones qui ne livrent pas le même mobilier : la partie sud de la nécropole, où se concentrent les tombes à mobilier exclusivement indigène, les cratères à pied et les décorations à cannelures, et la zone nord-est où les tombes à céramique uniquement. indigène sont également présentes mais avec un mobilier nettement moins abondant: les coupes à pied et les coupelles y sont bien attestées mais les cratères à pied et l'élément décoratif de cannelures sont absents.

L'étude de la répartition des autres catégories de mobilier permet de confirmer et de préciser sensiblement ces premières observations.

\section{Les CÉramiques Étrusques}

Le bucchero nero (fig. 6a). La carte de répartition des canthares en bucchero présente peu d'intérêt, ce mobilier étant présent dans presque toute la nécropole, à l'exclusion, toutefois, du secteur nord-ouest.

Les amphores étrusques (fig. 6b). Leur diffusion est autrement significative. Ce type de récipient est, en effet, totalement absent des parties sud et nordest de la nécropole. L'aire de dispersion des amphores étrusques délimite, en fait, une zone très homogène, de forme ovale qui occupe toute la partie centrale. Dans le détail, on relèvera que toutes les amphores appartiennent au type à lèvre en bourrelet; le seul exemplaire à col en forme d'amande se trouve, en revanche, très isolé dans la partie nordouest du gisement.

De façon plus générale, il apparaît enfin que les zones de répartition du bucchero nero et des amphores ne coïncident nullement : dans toute la partie sud et nord-est, où le bucchero est très bien attesté, les amphores étrusques sont totalement absentes. Une telle différence de répartition, mise en évidence sur des séries abondantes, ne saurait être fortuite et, devra donc être expliquée. Elle souligne cependant la faiblesse des méthodes de datation des sépultures basées sur les seules associations directes de mobilier.

\section{LES AMPHORES DE TYPE GREC (fig. 6c)}

Nous avons regroupé ici toutes les amphores à col cylindrique, corinthiennes, ioniennes ou ioniomassaliètes, faute d'une description détaillée de ce type de récipients. Là encore, la diffusion de ce type 

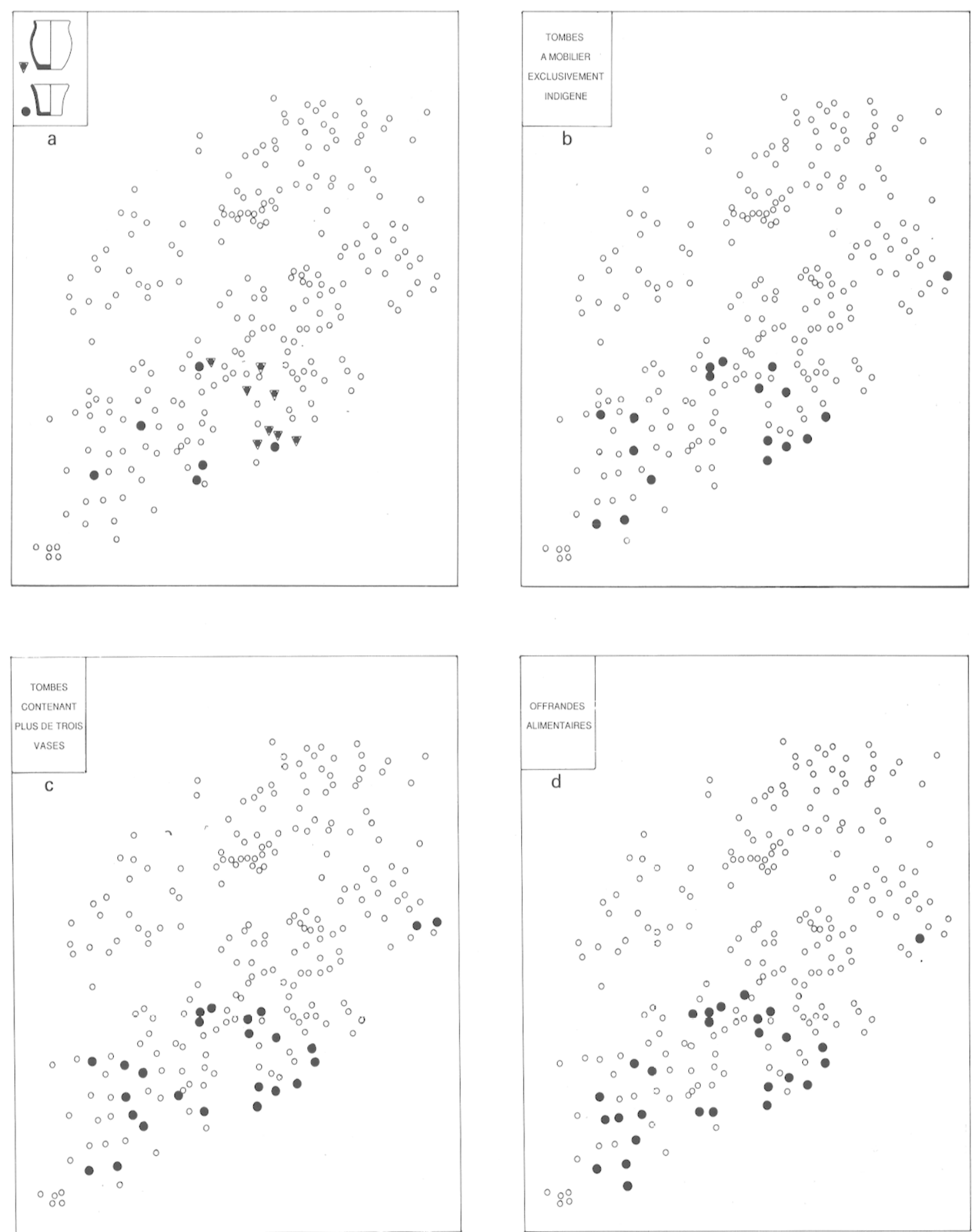

Fig. 5 - Cartes de répartition des gobelets tronconiques et ovoïdes (a), des tombes à mobilier exclusivement indigène (b), des tombes avec plus de 3 vases (c) et des tombes avec offrande alimentaire (d). 

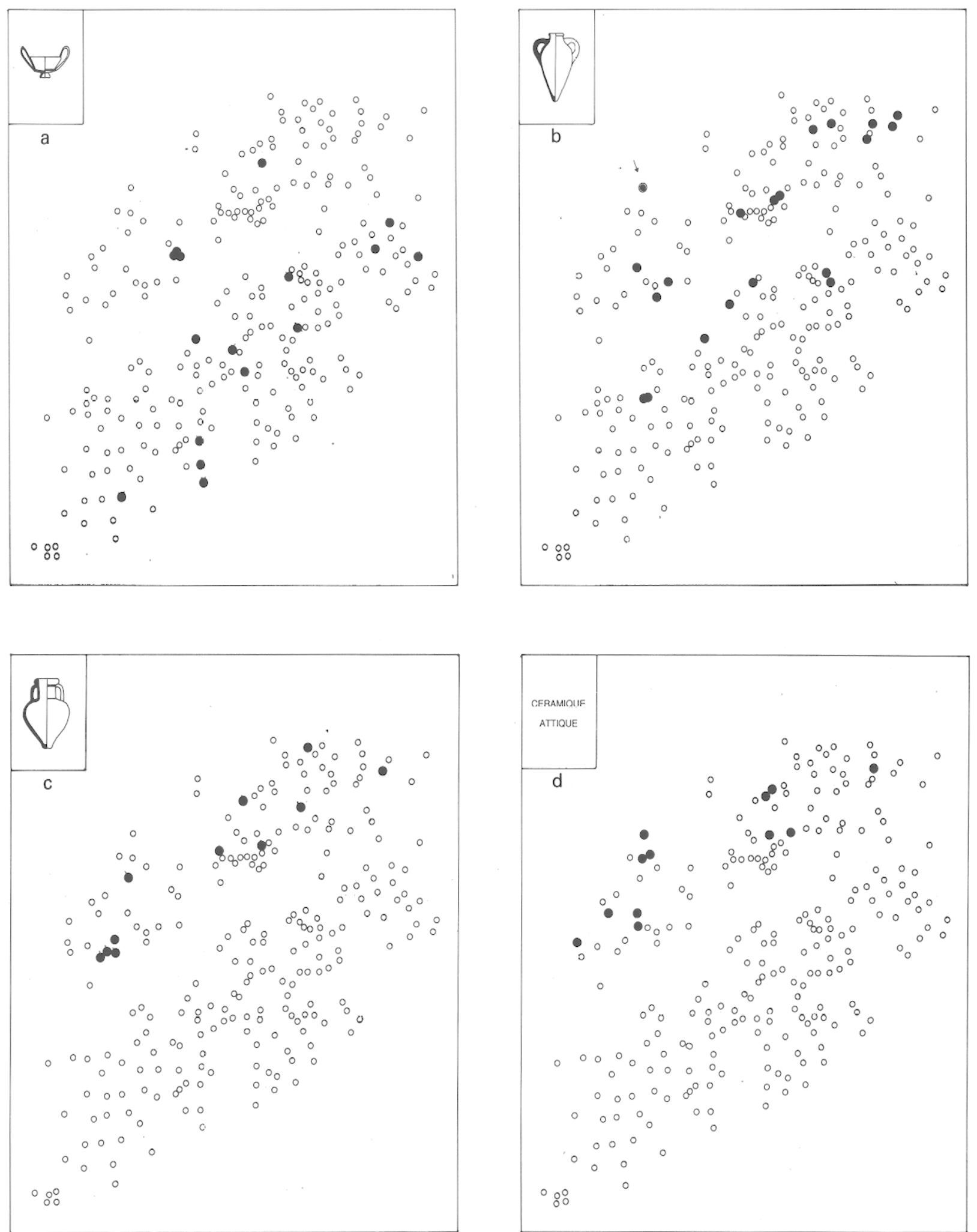

Fig. 6 - Cartes de répartition du bucchero nero (a), des amphores étrusques (b), des amphores de type grec (c) et des céramiques attiques (d). La flèche indique l'emplacement de la seule amphore étrusque à col en amande. 
d'objets est éloquente : tout ce mobilier est concentré dans la partie nord-ouest de la nécropole. Les zones sud et nord-est ne livrent, en revanche, que du mobilier très sporadique, résultant de charruages anciens. On notera d'ailleurs que les zones de répartition des amphores grecques et étrusques ne se chevauchent que très partiellement.

\section{Les Céramiques attiques (fig. 6d)}

Leur répartition vient renforcer les observations faites précédemment. La quasi-totalité des vases attiques est en effet regroupée dans une zone longitudinale de forme ovale dans les secteurs nord et nord-ouest de la nécropole. Leur aire de dispersion correspond très exactement à celle des amphores de type grec.

\section{LES CÉRAMIQUes À PÂTE CLAIRE}

Les stamnoi (fig. 7c) : les exemplaires attestés se trouvent tous dans les zones sud et nord-est de la nécropole.

Les ænochoés (fig. 7b) : si les œnochoés à simple décor de bandes sont présentes dans une très grande partie du gisement, les exemplaires décorés de rosettes, de points ou de larmes n'apparaissent que dans une zone limitée de la partie sud.

Les coupes ioniennes B2 à vernis noir ou à pâte claire en céramique grecque d'Occiderl (fig. 7a) : ce mobilier, dans lequel il aurait été intéressant d'opérer des subdivisions plus fines, n' est présent que dans la partie centrale de la nécropole ${ }^{11}$. Curieusement, son aire de répartition coïncide très exactement avec celle des amphores étrusques. Un seul exemplaire à vernis noir - est attesté dans la partie nord-ouest. En revanche, la zone sud, où pourtant abondent les œnochoés à pâte claire, n'a livré aucun récipient de ce type. Cette observation est à rapprocher de celles faites à propos du mobilier étrusque et invite, elle aussi, à beaucoup de prudence dans les tentatives de datation des tombes à partir des calégories de mobilier prises globalement.

\section{LA CÉramiQue Grise MONOCHROME}

Ce type de mobilier est très abondamment représenté. Il est assez bien connu grâce aux publications de J.-J. Jully. Les renseignements les

11 Les coupes à bord peint du type ionio-attique sont trop peu nombreuses et trop fragmentées pour pouvoir être utilisées. plus intéressants restent, cependant, ceux fournis par J. Giry qui a souvent donné les dimensions précises de ces récipients, permettant ainsi de les identifier en l'absence de dessins. Nous savons, en effet, grâce aux études typologiques qui ont été menées au cours de ces dernières années, que le caractère de production de série de ces céramiques permet souvent de les reconnaître à partir de leurs seules dimensions. La précision de ces indications nous permettra donc des études de répartition très fines.

Les cratères à pied haut (fig. 8a) sont présents dans toute la nécropole, à l'exception de la partie la plus méridionale. Leur très grande dispersion en fait un critère discriminant assez médiocre.

Les onochoés (fig. 8c) sont rares dans la partie sud de la nécropole, absentes des zones ouest et nordouest, mais bien attestées dans la partie centrale.

Les stamnoi (fig. 7d) sont trop peu nombreux pour que leur répartition soit vraiment significative. On relèvera cependant leur présence dans des secteurs très divers de la nécropole qui atteste la longue faveur de ce type de récipient.

Les coupelles hémisphériques (fig. 8d) sont totalement absentes des zones sud, ouest et nord-ouest de la nécropole et n'apparaissent en abondance que dans la partie centrale.

Les coupes de type $B 2$ à pâte grise (fig. 9d), trop peu nombreuses pour que leur diffusion soit significative, sont surtout présentes dans la partie centrale de la nécropole. Le seul exemplaire dépourvu d'anses se trouve, en revanche, à proximité de la zone nord-est.

Les urnes à col court et pied annulaire (fig. 9a) ont une répartition strictement localisée à la partie centrale de la nécropole.

Les gobelets carénés (fig. 8b) sont assez nombreux et bien connus par l'étude qui leur a été consacrée par J.-J. Jully et Y. Solier. Ils sont, pour l'essentiel, localisés dans la partie centrale de la nécropole. On relèvera avec intérêt l'apparition de quelques exemplaires au milieu de la zone sud.

Les plats à marli (fig. 9b) sont peu abondants et fragmentés. Ils sont très étroitement concentrés dans la partie nord.

Les décors ondés (fig. 9c). La carte de répartition des décors ondés est également très significative : la quasi-totalité est en effet concentrée dans la partie nord, dans un secteur qui coïncide assez précisément avec l'aire de diffusion des plats à marli. 

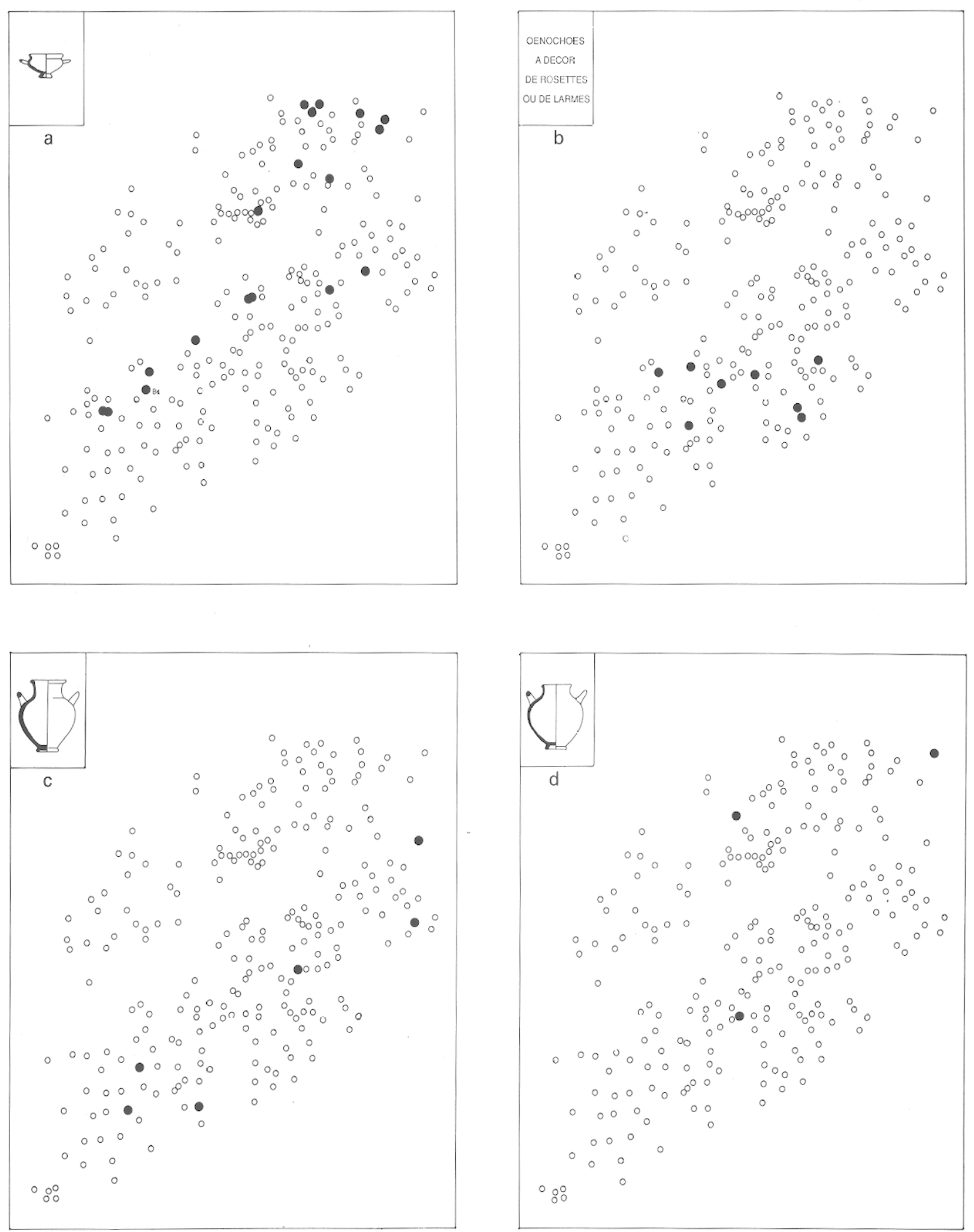

Fig. 7 - Cartes de répartition des coupes à pâte claire (a), des œnochoés à décor de rosettes ou de larmes (b), des slamnoi en céramique à pâte claire (c) ou en céramique grise (d). 

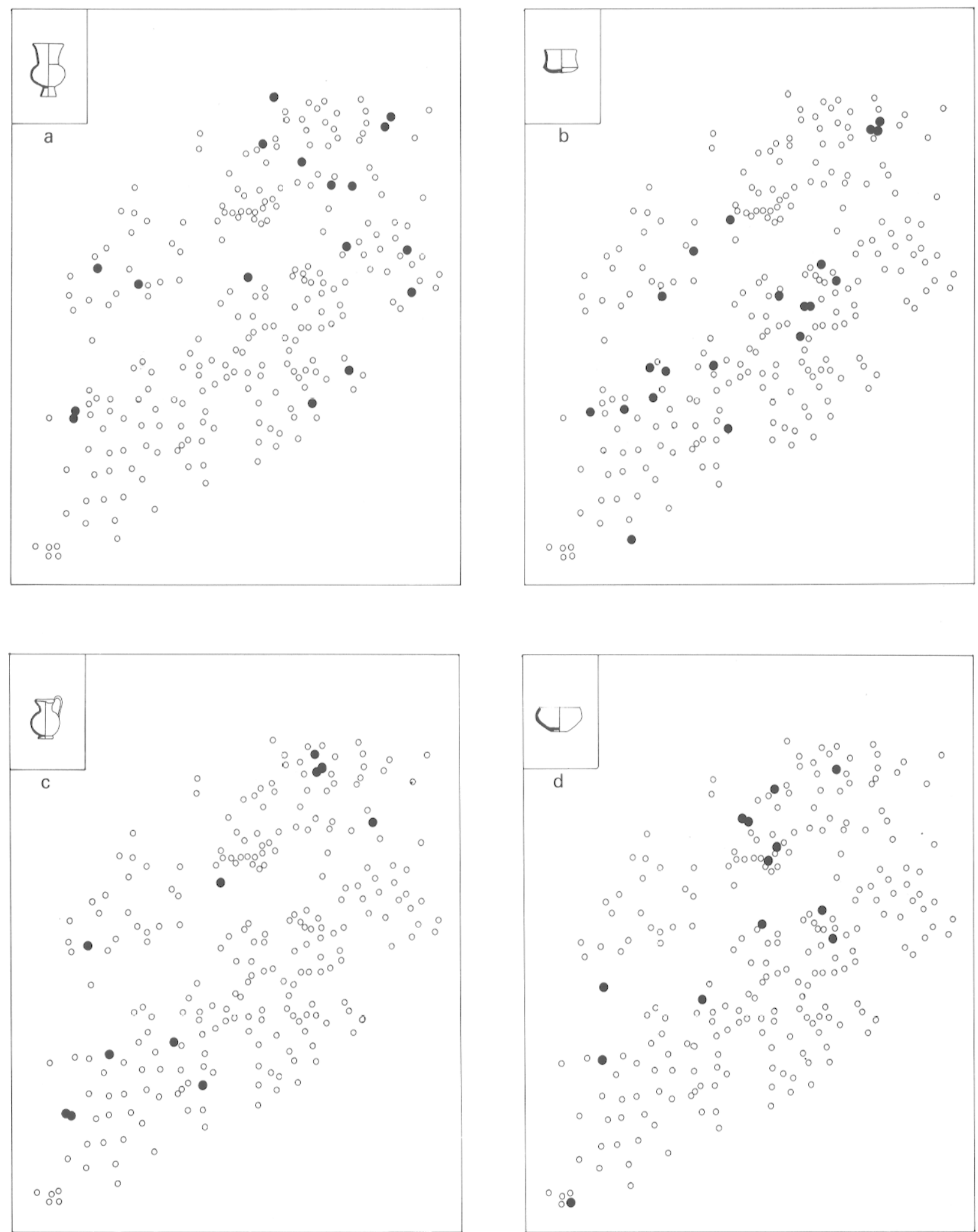

Fig. 8 - Cartes de répartition des céramiques grises : cratères à pied haut (a), gobelets carénés (b), œnochoés (c) et coupelles hémisphériques (d). 

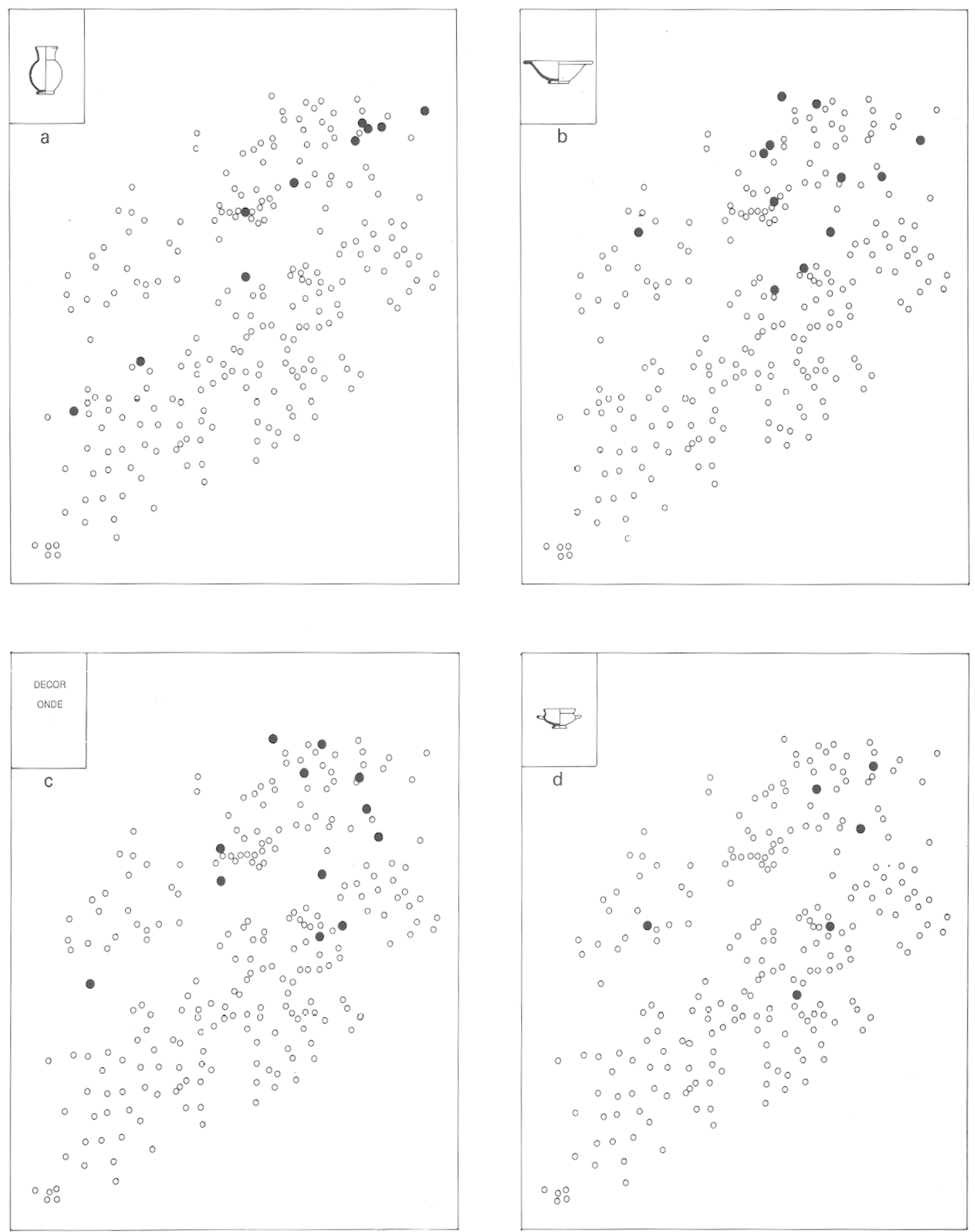

Fig. 9 - Cartes de répartition des céramiques grises : urnes à col court et pied annulaire (a), plats à marli (b), décors ondés (c) et coupes de type B2 (d). 


\section{LE MOBILIER MÉTALlique}

\section{Les fibules :}

- à ressort bilatéral court. Si les exemplaires en fer sont présents dans toute la nécropole, à l'exception toutefois de la partie la plus méridionale, les fibules en bronze à ressort bilatéral court n'apparaissent que dans la partie nord-est, pour devenir courantes dans le secteur central;

- à arbalète. Ce type de fibule presque exclusivement en bronze n'est attesté que dans les zones centrales et nord-ouest;

- de type navarro-aquitain. Elles sont trop peu nombreuses pour permettre une étude de répartition précise, on peut toutefois remarquer qu'elles sont absentes des zones sud et nord-est mais attestées dans les parties centrale et nord-ouest.

Les pointes de lance en fer. D'un point de vue typologique, on distinguera ici les pointes de lance longues (longueur supérieure à $28 \mathrm{~cm}$ ) et les pointes courtes. La répartition de ces deux types d'armes est assez remarquable. Les fers de grande taille ne se trouvent, en effet, que dans les secteurs sud-est de la nécropole (fig. 10). Les fers courts, en revanche, sont plus rares dans ces zones, mais caractérisent la partie centrale du gisement.

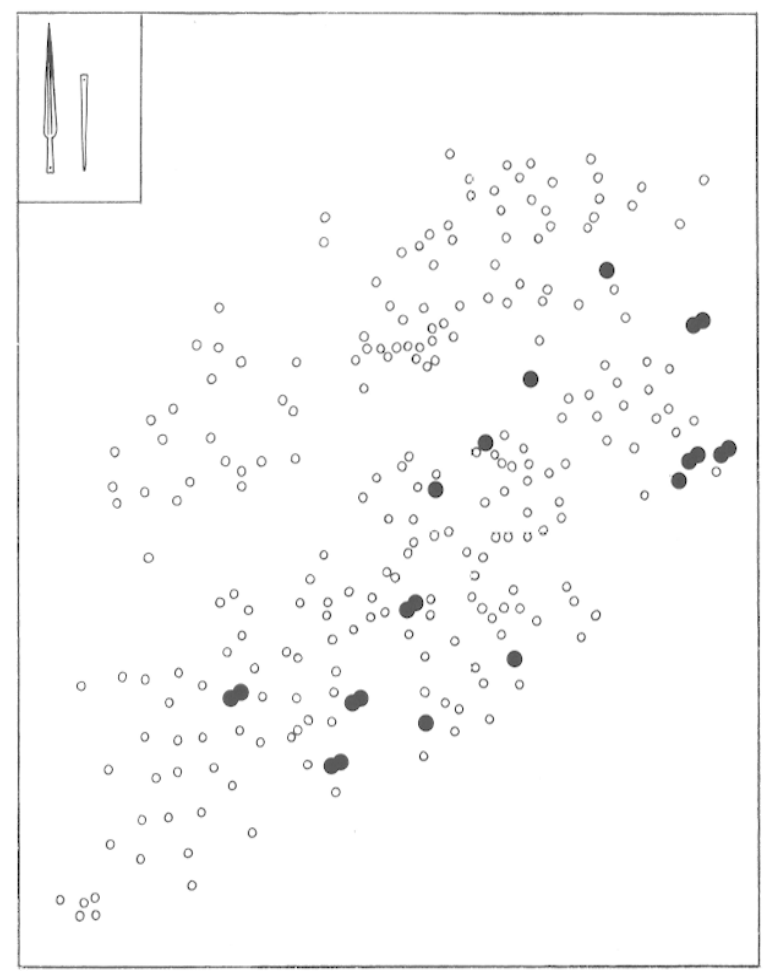

Fig. 10 - Carte de répartition des fers de lance de grande taille.
Les boucles de ceinturon (fig. 11c et d). L'aire de diffusion de ces pièces est, a priori, peu intéressante. La carte de répartition de la figure $11 \mathrm{c}$ montre, en effet, que les boucles à un seul crochet sont présentes dans toute la nécropole, à l'exception de la partie la plus méridionale. Il en va cependant différemment si l'on examine leur décoration qui permet de les ranger en deux grandes séries : à décor de fil martelé ou à décor de grènetis. On remarque que les premières sont toutes concentrées aux abords de la zone sud, alors que les exemplaires à décor de grènetis n'apparaissent que dans les zones centrale et nord-est de la nécropole (fig. 11c). Les exemplaires à deux crochets ne sont présents que dans la zone nord-est. Ils sont totalement absents de la zone sud, mais sont bien attestés, en revanche, dans la partie centrale (fig. 11d).

Les couteaux en fer (fig. 11b). Extrêmement nombreux, on les rencontre sur toute l'étendue de la nécropole. En l'absence d'indication détaillée sur la forme même de ces objets, nous avons essayé de les classer en fonction des seules données que nous possédions, c'est-à-dire leurs dimensions qui, elles, sont indiquées avec beaucoup de soin dans la publication de J. Giry. Si l'on a retrouvé des exemplaires de petite taille ou de taille moyenne dans toute la nécropole, il n'en va pas de même pour ceux de grande taille (longueur supérieure à $17 \mathrm{~cm}$ ) qui, eux, forment une série très homogène. On ne les trouve que dans les zones centrale et nord-ouest. Ils sont absents de la partie sud du gisement.

Les armilles en bronze (fig. 11a). Ces parures se concentrent de façon très remarquable dans une bande longitudinale de direction sud-ouest/nord-est. Un seul exemplaire se trouve à l'écart au sud.

\section{LES RITES FUNÉRAIRES}

Présence d'offrandes alimentaires (fig. $5 \mathrm{~d}$ ). La carte montre que les sépultures qui contiennent des offrandes alimentaires sont toutes concentrées dans la partie sud du gisement.

Importance du mobilier funéraire (fig. $5 \mathrm{c}$ ). Toutes les tombes qui comportent un mobilier funéraire abondant ont été recensées par opposition à celles qui ne comportent qu'un ou deux vases. Là encore, le contraste est très net entre les secteurs sud et nord-ouest de la nécropole. 

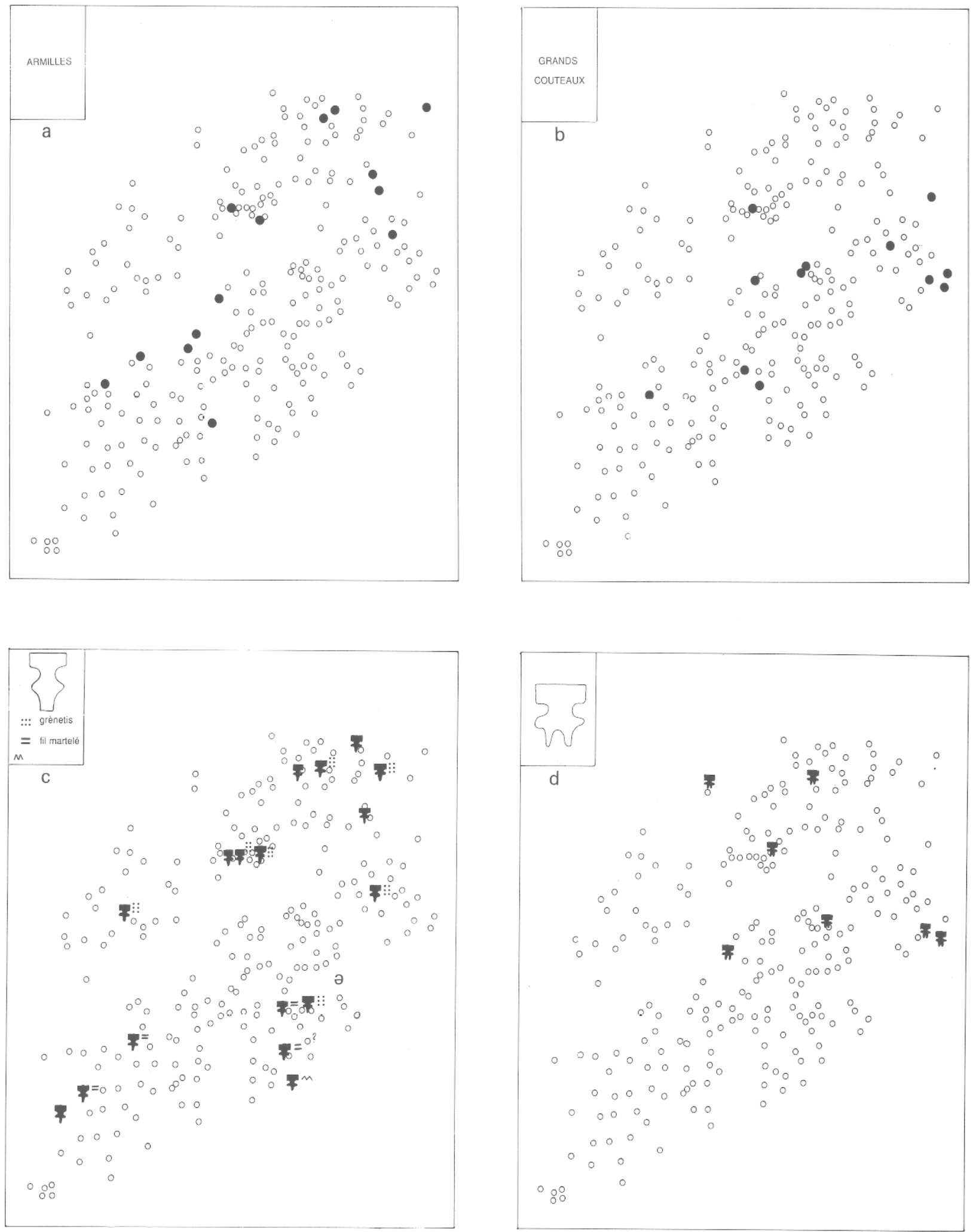

Fig. 11 - Cartes de répartition des armilles en bronze (a), des couteaux en fer (b) et des boucles de ceinturon (c et d). 


\section{LES RÉSULTATS}

\section{LA DÉLIMITATION DES ZONES D'ENSEVELISSEMENT}

I.es différences de répartition des diverses catégories de mobilier mises en évidence plus haut prennent toute leur signification lorsque l'on procède à une confrontation générale de toutes les cartes. On dispose, dès lors, non plus seulement d'informations partielles, valables pour un seul type de mobilier, mais d'indications multiples qui se recoupent. Cette manipulation se fait essentiellement par superposition des diverses cartes. Ce travail long et délicat permet de délimiter et d'opposer un certain nombre de zones qui ne livrent pas les mêmes catégories de mobilier et que nous allons essayer de caractériser avec plus de précision.

\section{LA ZONE I}

Elle correspond à la partie la plus méridionale de la nécropole et regroupe des tombes qui ont un certain nombre de caractères communs :

- ces tombes livrent toujours un nombre élevé de vases : 4 à 10 , exceptionnellement 13 ;

- les fosses contiennent presque toujours des offrandes alimentaires non brûlées;

- le mobilier est dans sa très grande majorité non tourné. Ses éléments les plus caractéristiques sont : les cratères ou urnes à pied haut ou mi-haut; les gobelets tronconiques à fond plat et les gobelets ovoïdes à lèvre resserrée; le décor de cannelures sur l'épaule des vases; les vases excisés qui, bien que très rares, se concentrent dans cette zone;

- les vases tournés sont peu répandus et ne représentent que $9 \%$ de l'ensemble des vases découverts en place dans les tombes de ce secteur. Ils se répartissent comme suit : 4 canthares en bucchero nero, soit $29 \%$ des céramiques tournées de ce secteur; un stamnos peint en céramique grise monochrome, très certainement importé $(7 \%$ des vases tournés); 9 vases à pâte claire, soit $64 \%$ des céramiques tournées de ce secteur ${ }^{12}$;

- l'absence de quelques séries de vases mérite d'être relevée : il s'agit essentiellement des amphores étrusques, des coupes ioniennes et de leurs imitations et de la céramique attique;

12 Les œnochoés hors contexte des tombes 12B, 179 et 198 ne modifieraient pas très sensiblement la répartition du mobilier fin (respectivement 24,6 et $70 \%$ ).
- le mobilier métallique est relativement abondant : les pointes de lance sont très longues et vont, en général, par paires; les seules fibules attestées sont en fer à ressort bilatéral court; les boucles de ceinturon sont rarissimes (un exemplaire attesté); les fibules à arbalète et les armilles en bronze sont inconnues dans cette zone.

Un examen très attentif de nos cartes de répartition permet d'affiner encore notre travail et de subdiviser cette zone en deux secteurs Ia et Ib (fig. 12). Cette distinction se fonde essentiellement sur la répartition des gobelets ovoïdes à lèvre évasée (fig. 5a), qui se concentrent tous dans la partie orientale de la zone I et définissent notre secteur Ib, par opposition au secteur Ia, où seuls les gobelets tronconiques à fond plat sont attestés. Des différences moins nettes mais néanmoins sensibles, comme l'adoucissement du profil des cratères, la diminution de la hauteur des pieds et l'apparition d'urnes à profil en $\mathrm{S}$ et décor d'incisions allongées - inconnues précédemment - contribuent à bien individualiser ce secteur Ib.

Pour le mobilier métallique, le secteur Ib voit se multiplier les fibules en fer à ressort bilatéral court qui étaient très rares en Ia. Notons enfin que la seule boucle de ceinturon de la zone I apparaît en Ib.

Tombes attribuées au secteur Ia : 189, 212, 231,233, 235, 236, 238, 239, 241, 242, 243, 246, 248, 249.

Tombes attribuées au secteur $\mathrm{Ib}: 11,12 \mathrm{~b}, 173,174$, 177, 178, 180, 181, 183, 190, 191, 192, 208.

\section{LA ZONE II}

Elle s'étend, pour l'essentiel, au nord-est du secteur Ib, mais comprend également quelques tombes isolées au sud et à l'ouest du secteur Ia. Les caractéristiques des tombes de ce secteur sont les suivantes :

- le mobilier céramique est en forte diminution : en règle générale, les tombes ne contiennent plus que 2 vases, l'ossuaire et un vase à boire, coupe, coupelle, gobelet ou canthare. Dans quelques rares cas, on note la présence d'un ou deux vases supplémentaires ${ }^{13}$;

13 Seule la tombe 98 , avec 9 vases, fait exception. Cette tombe est originale à plus d'un titre : le nombre élevé de vases et l'offrande alimentaire la rattachent à la zone I, le cratère à pied haut en céramique grise et la coupe-compotier la rapprochant plutôt de la zone II. 

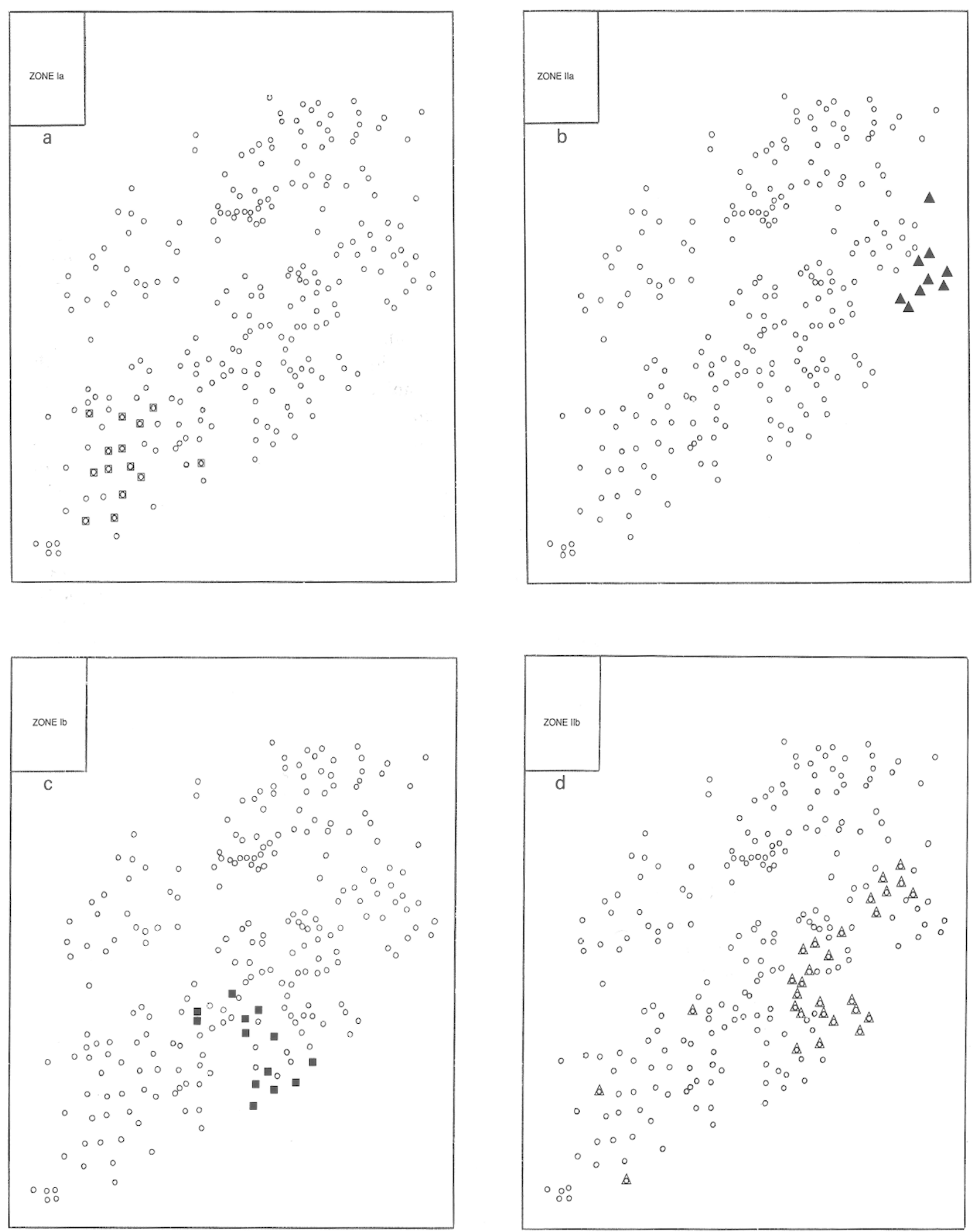

Fig. 12 - Localisation des tombes des zones I et II. 
- les offrandes alimentaires non brûlées disparaissent des fosses; cinq tombes en renferment encore : 98, 206, 244, 250 et $252^{14}$;

- le mobilier reste, en majorité, non tourné $(63 \%$ de la totalité du mobilier). Les formes attestées sont variées mais on note des changements sensibles : les cratères à pied haut ou mi-haut sont désormais rares et remplacés par des urnes à pied bas; un seul vase excisé est présent dans cette zone, contre quatre précédemment; les gobelets carénés, rares dans la zone $I$, sont désormais bien attestés; les coupelles à fond ombiliqué ne présentent aucun signe d'évolution, si ce n'est un façonnage plus lourd; le décor de cannelures a disparu;

- les vases tournés sont plus courants et se répartissent comme suit : $17 \%$ de bucchero nero (4 ou 5 canthares); $50 \%$ de céramiques à pâte claire : 3 stamnoi, 7 œnochoés, 2 coupes sans anse, une coupe-compotier, un couvercle de stamnos et une coupe B2 à vernis noir trouvée hors tombe, aux abords immédiats de la zone suivante; $23 \%$ de vases gris d'origine occidentale, 4 cratères à pied haut, 3 gobelets carénés, 2 urnes et un vase indéterminé;

- on relèvera à nouveau l'absence de quelques séries de vases : amphores étrusques, coupes ioniennes et leurs imitations et céramiques attiques;

- le mobilier métallique est abondant: les pointes de lance sont très inégalement réparties; la majeure partie de ces armes se concentre dans quelques tombes de la partie orientale de ce secteur; il s'agit presque toujours de fers très longs, déposés par paires; les fibules sont exclusivement de type à ressort bilatéral court en fer ou en bronze; l'originalité majeure de cette zone est l'apparition massive des boucles de ceinturon. Elles sont en majorité à un crochet mais deux exemplaires à deux crochets sont également attestés. On remarquera que tous les exemplaires à fil martelé se trouvent à proximité de la zone I.

Comme dans le cas de la zone I, un examen détaillé des cartes de répartition permet de distinguer deux secteurs, IIa et IIb, au sein de cette zone. Les tombes du secteur IIb s'individualisent par un nombre de vases légèrement plus élevé que dans la zone I Ia (3 à 5 vases au lieu de 2 ), l'absence de tout cratère à pied, même bas, et l'abondance des armes,

14 On remarquera qu'à l'exception de la tombe 252 toutes ces sépultures se trouvent à proximité immédiate de la zone I. Il s'agit de véritables tombes de transition qui permettent de dater le passage des fosses à vases d'accompagnement aux tombes à ossuaire complèté par le seul gobelet. notamment les pointes très longues. Deux types d'objets métalliques sont particulièrement caractéristiques de ce secteur : les agrafes de ceinturon à deux crochets et décor de grènetis et les grands couteaux en fer à virole et dos arqué. On peut ajouter que deux des trois stamnoi à pảte claire livrés par la zone II se trouvent dans le secteur IIb. On observera enfin que, d'un point de vue typologique, le secteur IIb est séparé du secteur IIa par une bande qui semble vierge de tombes.

Tombes attribuées au secteur IIa : 98, 108, 109, 112, $113,114,115,118,129,130,131,132,143,154,155$, $159,160,162,163,164,165,167,168,169,171,175$, 206, 244, 250.

Tombes attribuées au secteur IIb : 127, 128, 251, 252, 253, 254, 255, 256, 257.

\section{LA ZONE III}

Elle est très vaste (fig. 13). Elle occupe une bande longitudinale à l'ouest et au nord-ouest des zones I et II. Elle contient de nombreuses sépultures qui ont souvent été bouleversées par la charrue mais dont les caractéristiques principales peuvent être aisément dégagées :

- comme précédemment, il s'agit de tombes qui ne contiennent que peu de mobilier céramique. En règle générale, l'ossuaire n'est accompagné que d'un vase à boire, plus rarement par un vase supplémentaire qui peut servir de couvercle;

- les fosses sont toujours de petites dimensions, juste assez grandes pour accueillir l'ossuaire et son vase d'accompagnement;

- les offrandes alimentaires non brûlées sont totalement absentes dans cette zone.

Un certain nombre de différences dans la dispersion spatiale du mobilier permettent de subdiviser cette zone en trois secteurs bien individualisés :

Le secteur IIIa qui correspond à la partie centrale de la nécropole s'étend immédiatement au nord-ouest des zones I et II. Il se distingue par les caractères suivants :

- la céramique modelée ne représente plus que $17 \%$ de l'ensemble des vases de ce secteur: les cratères à pied et le décor de cannelures sont absents; les gobelets carénés sont bien attestés; les urnes et les coupelles sont nombreuses et variées;

- les vases tournés sont très abondants $(83 \%$ en comptant les amphores) : c'est dans ce secteur qu'apparaissent massivement les amphores étrusques; dix exemplaires au moins, à panse fuselée et petit col, sont attestés; une seule amphore ionio- 

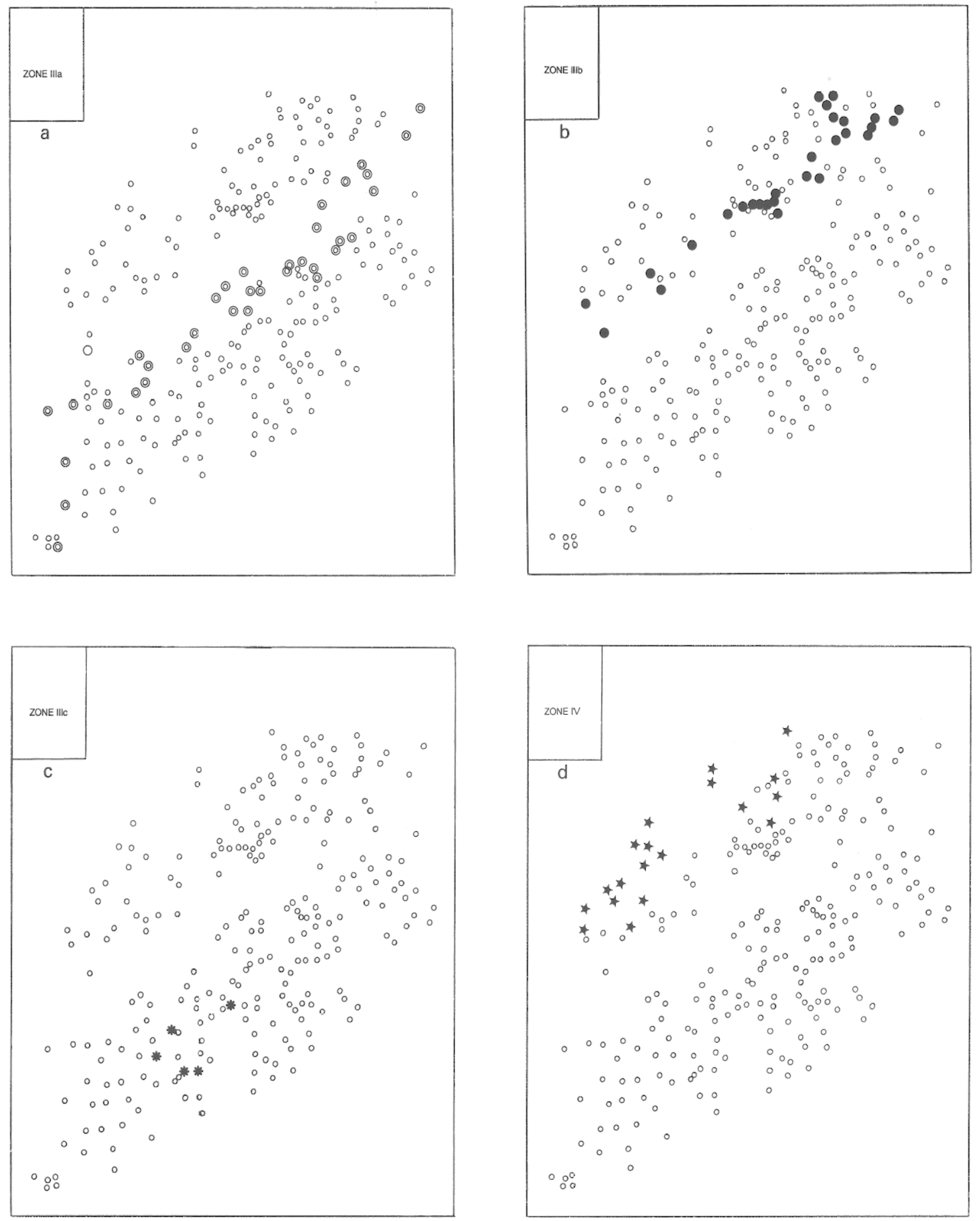

Fig. 13 - Localisation des tombes des zones III et IV. 
massaliète est signalée à la périphérie de ce secteur, mais il n'est pas certain qu'elle en fasse partie (T. 16/69);

- l'évolution est également très sensible pour les vases fins: les deux canthares en bucchero nero ne représentent plus que $3 \%$ de ce type de mobilicr; les vases à pâte claire sont en forte diminution avec $19 \%$; la forme la plus courante est la coupe ionienne de type $\mathrm{B} 1$ ou $\mathrm{B} 2$ à vernis noir ou d'imitation $(6$ vases sur 10); des œnochoés et des urnes à décor de larmes ou de bandes complètent le répertoire; les vases en céramique grise, avec $77 \%$, forment l'essentiel des vases fins; les formes sont désormais très variées : cratères à pied haut, urnes globulaires à col cylindrique et fond annulaire, œnochoés, gobelets carénés, écuelles à bords resserrés et un stamnos isolé. Cratères à pied et gobelets carénés restent les formes les plus courantes. Quelques plats à marli fragmentaires sont signalés dans la partie la plus proche du secteur suivant. Les décors ondés restent très exceptionnels; une seule jarre ibéro-languedocienne est attestée dans ce secteur; parmi les grandes séries de mobilier du vi's., seuls les vases attiques sont encore absents;

- le mobilier métallique est relativement abondant: les pointes de lance en fer sont en majorité courtes, mais quelques exemplaires de grande taille sont encore signalés; les fibules à ressort bilatéral court, en fer ou en bronze, restent courantes mais on voit apparaître les premières fibules à arbalète en bronze; les boucles de ceinturon sont rares, mais les seuls exemplaires attestés ont un décor de grènetis; la principale nouveauté est l'apparition massive des armilles en bronze qui caractérisent bien ce secteur.

Tombes attribuées à la zone IIIa : 7', 7", 8a, 8b, 15, $16 \mathrm{~A}, 16 \mathrm{~B}, 36,38,39,47,61,117,119,120,121,140$, $144,146,147,149,150,207,210,213,214,215,234$, $247,262,263,264,4 / 70,15 / 69,10 / 70,9 / 70,16 / 69$.

Le secteur $I I I b$ est séparé du précédent par une bande longitudinale de direction sud-ouest/nord-est vide de tombes. Il s'agit là très certainement d'un chemin protohistorique, d'une largeur que l'on peut estimer à environ $3 \mathrm{~m}$, qui a conditionné le développement de la nécropole durant cette période et qui, en particulier, sépare les secteurs IIIa et IIIb. Malgré leur très mauvais ètat de conservation, les caractéristiques des tombes de ce secteur peuvent être aisément mises en évidence :

- comme dans le secteur précédent, la céramique non tournée est très rare et atteint à peine $11 \%$ de l'ensemble des vases ${ }^{15}$. Le répertoire des formes s'est considérablement appauvri avec des urnes à profil en $\mathrm{S}$ très adouci, souvent décorées d'indentations au niveau du plus grand diamètre, des gobelets carénés, des coupelles hémisphériques ou tronconiques. Le façonnage maladroit trahit une fabrication hâtive et peu soignée;

- les céramiques tournées sont à nouveau abondantes et variées (89\%, amphores comprises) : les amphores restent en majorité étrusques mais cette zone se caractérise par l'apparition des amphores ionio-massaliètes, malheureusement toujours détruites par les labours ${ }^{16}$;

- la répartition statistique des céramiques fines est remarquablement constante : le bucchero nero, avec $4 \%$, reste peu représenté; les céramiques à pâte claire demeurent courantes et se répartissent à égalité entre des coupes ioniennes B2 à vernis noir, ou d'imitation, et des œnochoés. Les vases gris représentent, à nouveau, l'écrasante majorité du mobilier avec $77 \%$. Les formes sont les mêmes que précédemment : urnes globulaires, cratères à pied haut, gobelets carénés et coupelles à bords resserrés sont toujours les formes les plus courantes, mais on voit apparaître également des plats à marli, des imitations de coupe B2, une imitation de jarre ibérolanguedocienne. L'œnochoé paraît en revanche rare. Le décor ondé est bien attesté; comme précédemment, on peut signaler la présence d'une jarre ibérolanguedocienne à décor de bandes peintes; on signalera enfin, pour mémoire, la présence dans cette zone de quelques petits fragments isolés, toujours trouvés hors tombes, appartenant à des vases attiques des années 530-520 (coupe des Petits Maîtres, coupe de Cassel). Ce mobilier, découvert à proximité immédiate du secteur IV, a manifestement été dispersé et traîné par les labours;

- le mobilier métallique présente une évolution assez sensible: les boucles à un ou deux crochets cohabitent, mais seul le décor de grènetis est attesté. Les lances sont nettement plus rares que précédemment : il s'agit exclusivement de pointes courtes; les fibules à ressort bilatéral court et les fibules à arbalète coexistent; les armilles en bronze sont bien représentées comme dans le secteur précédent.

15 Les chiffres donnés ici sont nécessairement approximatifs mais l'ordre de grandeur est correct.

16 Les comptages que l'on peut faire à partir de l'inventaire de J. Giry montrent que, dans ce secteur, la part des amphores étrusques et grecques pourrait être respectivement de 60 et $40 \%$. 
Tombes attribuées au secteur IIIb : $2,3,4,6,34,35$, $41,42,14,45,46,49,68,72,122,123,134,137,138$, 216, 217, 221, 26' 266, 267, 270, 272, 4/69.

Le secteur IIIc est situé dans la partie sud de la nécropole, entre les secteurs Ia et Ib, dont il rompt en partie la continuité topographique. Il s'agit d'un secteur très perturbé par les labours, où de nombreuses tombes ont été bouleversées. Les quelques rares sépultures conservées et le mobilier dispersé révèlent la présence de deux types de tombes, les unes à mobilier abondant, d'un type comparable à celles $d u$ secteur Ib, et les autres à mobilier réduit au seul ossuaire et à son vase d'accompagnement. Les premières contenaient essentiellement des vases non tournés accompagnés d'onochoés à rosettes de points; le mobilier métallique comprend les habituelles pointes de lance longues et courtes et une boucle de ceinturon simple à nervure. Les secondes ont livré des vases gris, cratères à pied haut, œnochoés, un canthare en bucchero nero el des vases non tournés. Le mobilier métallique comprend notamment un javelot en fer et deux fibules à arbalète. Il rappelle très précisément celui livré par le secteur IIIb auquel il convient de rattacher ces tombes à ossuaire et vase d'accompagnement isolé.

Ce secteur IIIc correspond donc à une véritable enclave de tombes plus récentes au milieu de la zone I, qui au départ, devait s'étendre de Ia vers Ib sans solution de continuité, comme le prouvent les quelques vestiges de tombes anciennes qui subsistent au sein de cette enclave. L'installation de ces tombes récentes au milieu d'une zone d'ensevelissement ancienne, qui manifestement n'est pas respectée, mérite de retenir toute notre attention.

Tombes appartenant au secteur IIIc : 12A, 40A, 184, $185,187,193,196,197,199,200,201,202,203,204$, 205,219 .

Tombes du secteur IIIc à rattacher à la zone I : 10A, 185 ?, 193, 199, 200, 202.

Tombes du secteur IIlc à rattacher à la zone IIIb : 12A, 187, 203, 205, 197.

\section{LA ZONE IV}

Elle coincide avec la partie nord-ouest de la nécropole. Les tombes sont, là encore, très détruites et aucune étude de répartition sérieuse n'est possible. L.es limites exactes de ce secteur restent donc assez floues. Pour autant que l'on puisse en juger, cette zone présente les caractéristiques suivantes :

- d'assez nombreuses tombes ne possèdent plus d'ossuaire; le loculus traditionnel est souvent remplacé par une grande fosse de plusieurs mètres carrés dans laquelle s'entassent pêle-mêle des fragments de vases brisés, des éléments métalliques cassés ou fondus, des esquilles d'os et des pierres rubéfiées ${ }^{17}$. Dans le détail, on nole que des lombes avec ossuaire subsistent dans les parties nord-est et sud de ce secteur et que le nouveau rite ne s'imposera totalement que dans la partie la plus occidentale du secteur;

- le mobilier est très fragmenté et souvent abondamment remanié par les labours de sorte qu'aucun comptage n'est possible. On notera simplement de façon très globale ses principales caractéristiques: l'élément le plus frappant est l'apparition massive des céramiques attiques du dernier quart du $\mathrm{VI}^{e} \mathrm{~S}$. : coupes à yeux prophylactiques, coupe à vernis noir de lype $\mathrm{C}^{\mathbf{1 8}}$; les amphores semblent en majorité de type ionio-massaliète, mais les exemplaires étrusques sont encore bien altestés. On notera, en particulier la présence d'une amphore à lèvre en amande, la seule de ce type signalée dans la nécropole ${ }^{19}$; les céramiques grises restent très abondantes avec les habituels cratères à pied haut, gobelets carénés, plats à marli, urnes, coupelles à bords rentrants; les céramiques à pâte claire sont représentées par des œnochoés, des coupes d'imitation $B 2$ et des coupes ionio-attiques inspirées des coupes à bandes attiques ${ }^{20}$; le bucchero nero, avec au moins deux exemplaires, n'est pas négligeable; la céramique non tournée reste rare;

- le mobilier métallique est peu caractéristique : rares pointes de lance toujours courtes, rares fibules à ressort bilatéral court, remplacées par des fibules à arbalète et des fibules de type navarro-aquitain, boucles à un ou deux crochets à décor de grènetis.

Tombes attribuées au secteur IV : 1/69, 2/69, 5/69, $13 / 69,14 / 69,7 / 69,8 / 69,9 / 69,12 / 69,10 / 69,11 / 69$, 8/70, 135, 226, 227, 228, 232, 273, 274.

17 Pour le détail, je renvoie à la description de Chr. Llinas et A. Robert, loc. cit., p. 17-24.

18 Ce mobilier est très soigneusement décrit par J.-J. Jully, Céramiques grecques ou de type grec ..., op. cit., p. 736741.

19 B. Liou, Note provisoire sur deux gisements grécoétrusques (Bon Porte 1 et pointe du Dattier), Cahiers d'Archéologie Subaquatique, III, 1974, p. 7-19. L'auteur donne le dessin de cette amphore ainsi que le dessin de l'amphore ioniomassaliète de la tombe $7 / 69$.

20 Pour des vases comparables, voir A. Nickets et P.-Y. Genty, Une fosse à offrandes du vie s. av. J.-C. à La Monédière, Bessan, Hérault, Revue Archéologique de Narbonnaise, VII, 1974, p. 25-57. 


\section{La DATATION DES PHASES DE DÉvelopPEMENT DE LA NÉCROPOLE}

L'étude détaillée des aires de répartition des diverses catégories de mobilier permet donc de subdiviser la nécropole en huit secteurs regroupés en quatre grandes zones. Il convient maintenant d'en préciser la chronologie. Conformément à un principe que j'ai déjà exposé, je dégagerai en priorité leur chronologie relative, avant de les replacer dans le cadre d'une chronologie absolue. Il est en effet clair que si les progrès de notre connaissance du mobilier peuvent permettre d'affiner la datation de nos zones, ils n'affecteront en rien leur position relative.

\section{LA SUCCESSION DES ZONES D'ENSEVELISSEMENT}

J'ai rappelé que l'existence, dans une nécropole, d'un certain nombre d'aires de diffusion limitées et bien individualisées pouvait avoir une double signification, soit ethnologique (groupement par sexe, par famille, etc.) soit chronologique. Dans notre cas, il apparait clairement qu'il s'agit d'une succession chronologique. On constate, en effet, que les diverses catégories de mobilier retenues comme critères d'étude se retrouvent à peu près toujours dans des zones topographiquement voisines, mais avec des représentations différentes. Les variations, diminutions ou augmentations, sont régulières lorsque l'on passe d'une zone à l'autre et affectent toujours plusieurs critères qui varient dans le même sens. Sauf dans un cas, qu'il nous faudra d'ailleurs expliquer, il n'y a pas de véritable rupture dans les variations de représentativité des critères : tel type de céramique, très fortement représenté dans une zone, apparaîtra, en règle générale, nettement moins bien attesté dans des zones immédiatement contiguës. Ces variations correspondent évidemment aux phases d'apparition, d'utilisation régulière et enfin de disparition des diverses catégories de mobilier. Si, de façon globale, l'on prend en compte l'ensemble des critères, il apparaît clairement que l'évolution se fait toujours dans le même sens : il faut donc en conclure que le rapport entre les diverses zones que nous avons délimitées est bien d'ordre chronologique.

L'examen de notre collection de cartes montre que les zones d'ensevelissement se succèdent dans l'ordre suivant :

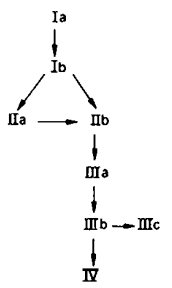

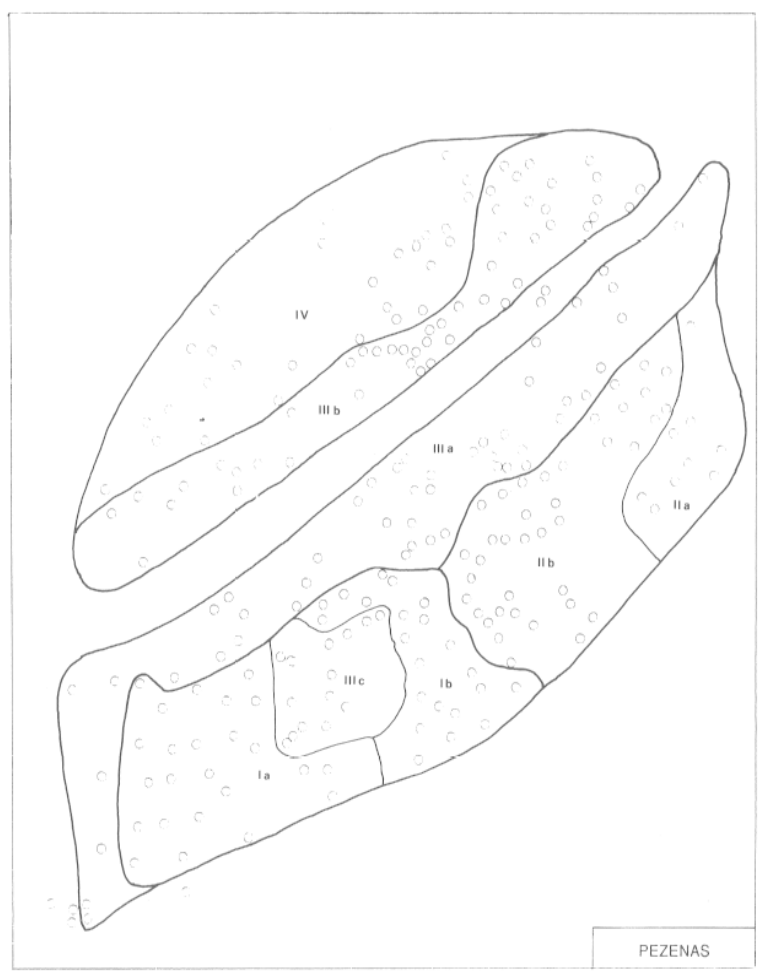

Fig. 14 - Développement topographique de la nécropole de Pézenas.

La carte de localisation des diverses zones (fig. 14) est très éloquente : elle montre, en premier lieu, que la nécropole a connu, à partir d'un noyau primitif situé en Ia, un développement général du sud-ouest vers le nord-est, de Ia vers Ib, I Ia et IIb, puis de là en direction du nord-ouest, de IIa et IIb vers IIIa, IIIb et enfin IV. Elle révèle également que ce développement n'a pas été régulier et comporte au moins deux "accidents» majeurs, deux ruptures qu'il nous faut expliquer :

- la première, en fait la seconde chronologiquement, se place entre les secteurs Ia et $\mathrm{Ib}$, où vient s'intercaler un groupe de tombes - que nous avons appelé IIIc - et dont le mobilier rappelle celui du groupe IIIb. Nous avons donc là un cas d'installation d'un groupe de tombes récentes au milieu d'une zone plus ancienne dont elle rompt la continuité topographique ;

- la seconde rupture apparaît au niveau des secteurs IIa et IIb : comme nous l'avons vu, les tombes du secteur IIb forment un ensemble limité, mais homogène et très original. Ce groupe de tombes présente en effet des caractères qui le rapprochent des zones anciennes Ia et Ib : tombes à mobilier en majorité - sinon en totalité — non tourné, vases relativement abondants, offrande alimentaire, présence de stamnoi à pâte claire, de pointes de lance 
longues, mais aussi un certain nombre d'éléments qui rappellent la zone IIa, boucles de ceinturon, cratères à pied haut en céramique grise. En revanche, il se distingue fortement des secteurs Ia et Ib par l'absence des cratères non tournés et des décors de cannelures. Son originalité par rapport au secteur I Ia est également très nette, en particulier au niveau du mobilier métallique. I.es boucles de ceinturon sont en effet ici à deux crochets, mais surtout on voit apparaître un type de grand couteau à virole et dos arqué qui était totalement inconnu dans les phases précédentes. On insistera, enfin, sur le fait que ce secleur se trouve à l'écart de l'aire de développement primitive de la nécropole, comme si l'on avait voulu isoler un groupe de tombes.

D'un point de vue chronologique, ce groupe ne peut être placé plus bas que la phase de transition entre les zones I et II, à un moment où les sépultures contiennent encore un mobilier indigène abondant et conservent parfois les restes d'une offrande alimentaire. La très grande originalité du faciès mobilier de ce groupe et sa situation à l'écart, peuvent surprendre mais s'expliquent pourtant aisément si l'on admet que ces deux faits sont liés et s'éclairent mutuellement. Il suffit, en effet, d'admettre que nous avons affaire ici à un nouveau noyau de développement de la nécropole, d'un faciès original, topographiquement indépendant, mais en partie contemporain des zones de développement précédentes. Il s'agit donc là d'un moment important dans le développement de notre cimetière, dont il nous faudra comprendre la signification.

A partir de la zone II, la nécropole s'étend ensuite au secteur IIIa, puis au secteur IIIb, ces derniers s'agençant, comme nous l'avons vu, par rapport à une bande longitudinale, vierge de tombes, dans laquelle nous n'hésiterons pas à reconnaître un chemin, simple voie de desserte de la nécropole ou ancien chemin que les tombes ont respecté. Dans sa dernière phase, la nécropole s'étend enfin dans la zone IV qui correspond à la partie nord-ouest du gisement.

\section{ÉLÉMENTS DE CHRONOLOGIE ABSOLUE}

Le nombre et la qualité des vases importés découverts dans la nécropole de Pézenas confèrent à ce gisement une valeur exceptionnelle pour la datation de toutes les céramiques fabriquées en Gaule méridionale. Pour conserver à ces associations leur valeur et pour pouvoir, à l'avenir, procéder à des comparaisons avec d'autres gisements régionaux, il était indispensable d'asseoir notre chronologie uni- quement sur des documents datés hors de Gaule méridionale : coupes et œnochoés de Grèce de l'Est, bucchero nero, céramiques attiques. Ces diverses séries permettent de dater nos différentes phases de développement avec une précision très remarquable.

La zone IV a livré une série très homogène et bien datée de coupes à yeux à figures noires et de coupes à vernis noir de type $C$ qui se placent globalement entre 530 et les premières années du $\mathrm{V}^{\mathrm{e}} \mathrm{s}^{21}$. Une seule sépulture $(11 / 69)$ contient un mobilier plus ancien, appartenant à la première moitié du $\mathrm{vI}^{\mathrm{e}} \mathrm{s}$., mais qui n'infirme pas la datation d'ensemble retenue pour cette zone ${ }^{22}$.

Si la période de première utilisation de cette zone doit être placée autour des années 530-520, son abandon, qui coïncide avec la fin de la nécropole, peut être situé dans les années 490-480. Une telle précision aurait de quoi surprendre si nous n'avions ici un mobilier abondant où ne figure aucun élément postérieur à cette date. L'exploration de vastes aires vierges de tombes à la périphérie de cette zone IV indique, d'ailleurs, que les limites de la nécropole ont bien été atteintes. Cet abandon de la nécropole dans les premières années $d u v^{e} s$. mérite d'être relevé et pourra être mis en rapport avec d'autres discontinuités relevées, par exemple, dans les habitats.

La zone III se caractérise par l'utilisation massive des coupes ioniennes de type $\mathrm{B} 2$ à vernis noir el de leurs imitations locales. Au nord-ouest, au contact de la zone IV, on rencontre quelques fragments isolés de coupes attiques des années 530-520. En revanche la zone II, qui s'étend au sud-est, n'a livré qu'une seule coupe B2 à vernis noir, d'ailleurs retrouvée à proximité immédiate de la zone $\mathbf{I I I}$. La zone III peut donc globalement être datée des années 570-520. Les deux secteurs IIIa et IIIb ayant une surface et un nombre de tombes comparables, nous proposons de dater le premier des années 570550 et le second des années 550-520. L'enclave du secteur IIIc, qui a livré un mobilier proche de celui du secteur IIIb, doit donc être datée également de la même période $550-520$.

La zone II est globalement beaucoup plus difficile à dater que les précédentes. Si l'absence des coupes ioniennes de type B2 - à l'exception de l'exemplaire à vernis noir, trouvé hors tombe indique un horizon antérieur à 580 , rien ne permet de

21 J.-J. Jully, Céramiques grecques ou de type grec..., op. cit. p. 736-741.

22 On peut, en effet, penser à une tombe plus ancienne volontairement placée à la périphérie de la nécropole. 
dater avec précision le commencement d'utilisation de cette zone. On peut tout de même noter que le nombre de tombes, la surface occupée et la présence de phases plus anciennes, qui livrent également du mobilier des débuts du $\mathrm{VI}^{\mathrm{e}}$ s., interdisent de placer les premières tombes de ce secteur plus haut que 590 . C'est donc une datation générale dans les années 590-570 que nous adopterons. Ces limites chronologiques conviennent parfaitement pour le bucchero nero et les céramiques à pâte claire de ce secteur, œnochoés à bandes, slamnoi et coupes profondes sans anses. Dans ces conditions, la zone IIa, qui regroupe les tombes les plus anciennes de ce secteur peut être datée des années 590-580.

La zone I rassemble les tombes les plus anciennes de la nécropole. L'absence totale des vases les plus caractéristiques du faciès Grand Bassin I, comme les coupes à profil convexe-concave et les urnes à épaule carénée, dont l'utilisation est encore bien attestée autour des années 620-610 dans les nécropoles voisines d'Agde ${ }^{23}$ et de Servian ${ }^{24}$, interdit de placer les tombes les plus anciennes de Pézenas avant cette date. Nous situerons donc la phase primitive de notre nécropole dans la période 610-590, ce que ne contredisent ni les stamnoi à pâte claire ni les œnochoés à rosettes inspirées du corinthien ancien, ni le canthare en bucchero à décor de filets et de chevrons. Faute d'éléments plus précis et compte tenu du nombre sensiblement égal de tombes, nous daterons les deux phases Ia et Ib mises en évidence par nos cartes de répartition de façon arbitraire : 610-600 pour Ia, 600-590 pour Ib.

En conclusion, je rappellerai d'abord que l'époque de la première utilisation de cette nécropole nous est bien connue. Mon analyse de son développement topographique et l'absence, dans la phase la plus ancienne, des fossiles directeurs les plus caractéristiques des nécropoles du vir ${ }^{\mathrm{e}} \mathrm{s}$. montrent que les débuts de ce cimetière se placent indiscutablement dans les toutes dernières années du vil ${ }^{e}$ s. L'abondance relative des tombes anciennes, toutes situées dans un horizon chronologique très court, et la très grande homogénéité du mobilier indiquent que l'on a affaire non pas à un petit noyau primitif qui se serait progressivement développé, mais au contraire à un groupe important et bien constitué dont nous n'avions précédemment aucune trace. L'absence

23 A. Nickels, G. Marchand et M. Schwaller, Agde, la nécropole du premier Age du Fer, Paris, 1989.

24 J.-L. Espérou, A. Nickels et P. Roques, La nécropole du I ${ }^{\text {er }}$ Age du Fer de "La Cartoule" à Servian, Hérault, Archéologie en Languedoc, 3, 1980, p. 93-102. totale de tombes des phases les plus anciennes de l'Age du Fer atteste d'ailleurs un hiatus important entre notre première phase d'ensevelissement et les quelques tombes plus anciennes attribuées au Chalcolithique ou à l'Age du Bronze qui ont été découvertes à l'emplacement de notre nécropole.

Bien sûr, rien ne permet d'affirmer qu'il n'existait pas, à proximité, une nécropole plus ancienne. La position topographique de la phase I - en grande partie enclavée dans des zones de développement plus récentes - montre cependant que même si un tel cimetière avait existé, la rupture de continuité avec nos tombes les plus anciennes serait néanmoins totale. Grâce aux fouilles menées par J. Giry sur l'oppidum de Saint-Siméon ${ }^{25}$, qui correspond à notre nécropole, nous savons que tout le mobilier datable livré par cet habitat appartient au $\mathrm{VI}^{\mathrm{e}} \mathrm{s}$. En raison même de son importance, on peut considérer que la nécropole de Saint-Julien est la seule zone funéraire de ce site. L'exacte contemporanéité que l'on relève entre le mobilier livré par la nécropole et celui fourni par l'habitat confirme que nous sommes en présence d'un habitat de hauteur important qui se développe très rapidement à partir des dernières années du $\mathrm{vI}^{\mathrm{e}} \mathrm{s}$. On rappellera que c'est très exactement à la même période que se place la première occupation d'un autre habitat très connu de la basse vallée de l'Hérault, La Monédière à Bessan, qui s'étend sur une terrasse alluviale du fleuve à une douzaine de kilomètres au sud de Pézenas. Nous avons là deux témoignages des nouvelles relations - ou peut-être des nouveaux rapports de force - qui s'établissent dans la basse vallée de l'Hérault à l'extrême fin du vir s. avant notre ère.

La communauté indigène installée sur la colline de Saint-Siméon est dès le départ en contact direct ou indirect avec les commerçants grecs ou étrusques, comme en témoignent les importations de céramiques - stamnoi, œnochoés et canthares - retrouvées dans les tombes de la zone I. La rareté et la concentration de ces vases dans quelques tombes à mobilier abondant montrent que nous sommes là dans une nouvelle phase d'exploration, distincte de celle que la vallée de l'Hérault a connue dans le troisième quart du $\mathrm{vII}^{\mathrm{e}} \mathrm{s}^{26}$, mais où les échanges restent encore limités aux élites. Durant ces périodes

25 J. Giry, L'oppidum Saint-Siméon, commune de Pézenas, Eludes sur Pézenas et sa région, 1970, 2, p. 3-6.

26 A. Nickels, C. Pellecuer, G. Raynaud, J.-C. Roux et M. AgdÉ, La nécropole du I ${ }^{\text {er }}$ Age du Fer d'Agde : les tombes à importations grecques, MEFRA, 93, 1981, 1, p. 89-125. 
anciennes, seuls les vases de luxe sont déposés dans les tombes: c'est ce qui peut expliquer l'absence, dans les tombes de Pézenas, de toute amphore étrusque jusque vers les années 570, alors que ce mobilier est bien attesté dès les débuts du $\mathrm{vI}^{\mathrm{e}} \mathrm{s}$. dans un sile de la basse vallée de l'Hérault comme Bessan.

On notera qu'à la différence de ce qui se passe dans d'autres régions - l'arrière-pays nimois par exemple ${ }^{27}$ - rien n'autorise ici à conclure à une quelconque antériorité du commerce étrusque sur le commerce grec. Même dans les phases les plus anciennes de la nécropole, les vases en bucchero ne représentent que $30 \%$ de la totalité des vases fins, le reste ctant constitué de vases à pâte claire, pour la plupart originaires de Grèce de l'Est. Les récentes tentatives pour attribuer aux Etrusques le monopole de la distribution des céramiques grecques archaïques dans l'ensemble du Midi méditerranéen ne résistent donc pas à l'analyse pour le secteur qui nous intéresse.

L'une des caractéristiques des tombes languedociennes du $\mathrm{VI}^{\prime \prime} \mathrm{s}$. est l'apparition régulière des armes. En cela, elles s'opposent fortement aux tombes du $v_{I I}$ s. qui sont pratiquement toujours dépourvues de ce type d'objel. J'ai longtemps pensé ${ }^{28}$, comme d'autres $^{29}$, que cette apparition soudaine des armes pouvait s'interpréter comme la réponse indigène à la menace - réelle ou supposée - que pouvait représenter la fréquentation des côles du Languedoc par les navigateurs grecs ou étrusques. L'étude de la nécropole de Pézenas m'invite aujourd'hui à nuancer quelque peu ce point de vue. On constate, en premier lieu, que les armes apparaissent à Pézenas dès l'époque de la première utilisation de la nécropole. Aux phases I et II, soit entre 610 et 590 , près de $25 \%$ des tombes contiennent déjà des armes : dès les environs de 600, un homme sur deux est donc un guerrier, ou du moins, se fait enterrer avec les attributs du guerrier, alors qu'au vir s. seule une tombe sur cent contenait des armes. La mutation est donc à la fois rapide et profonde. L'examen attentif des armes découvertes dans les tombes anciennes de Pézenas est également très instructif. On constate, en effet, que ces armes sont, pour la plupart,

27 M. PY, La Liquière à Calvisson, Gard. Village du Ir Age du Fer en Languedoc oriental, Paris, 1984, p. 263-264.

28 A. Nickels, Les Grecs en Gaule : l'exemple du Languedoc, in: Modes de contacts et processus de lransformation dans les sociétés anciennes, Actes du colloque de Cortone, PiseHome, 1983, p. 409-425.

29 M. Louls, O. et J. Taffanel, Le Premier Age du Fer languedocien, III, Bordighera-Montpellier, 1960, p. 401. d'origine très probablement grecque ou étrusque. C'est évidemment le cas des cnémides mais également, sans doute, de pièces dont l'origine est plus difficile à déterminer, comme les cuirasses ou les lances. Ces dernières appartiennent, en effet, à un type très particulier, à fers très longs et effilés, qui est totalement inconnu dans le monde indigène au $\mathrm{vII}^{\mathrm{e}} \mathrm{s.}^{30}$ où les deux seules pointes présentes en milieu funéraire sont d'un type très court. Ce type d'arme est, en revanche, bien attesté à cette époque dans le monde grec et tout porte à croire que les exemplaires livrés par la nécropole de Pézenas ont été fournis par les nouveaux arrivants, Grecs ou Etrusques. Les éléments de cuirasse retrouvés dans certaines tombes ont, très probablement, la même origine. Dans ces conditions, ce ne sont pas des éléments disparates d'armement grec que livrent les tombes les plus anciennes de Pézenas, mais bien des panoplies complètes associant la cuirasse, les cnémides, les deux lances à fers très longs et exceptionnellement l'épée. Rien n'interdit d'ailleurs de penser que cet équipement comprenait également le bouclier rond, qui a pu entièrement disparaître sur le bûcher. Qu'il s'agisse d'un armement de parade ou d'un équipement réellement destiné au combat importe peu : dès les premiers contacts, les nouveaux arrivants fournissent aux indigènes des armes offensives et défensives de toute première qualité.

Cette apparition des armes dans les tombes peut ètre interprétée de diverses manières. La plus immédiate consiste à considérer que ces objets font simplement partie des échanges normaux avec les nouveaux arrivants, qu'il s'agit de simples éléments de commerce qui n'ont guère plus de signification que les œnochoés ou les stamnoi que l'on trouve dans les tombes à la même époque. Dans cette hypothèse, ils témoigneraient simplement de l'enrichissement des élites ${ }^{31}$; objets de luxe, ils se retrouveraient tout naturellement dans les tombes. Cette explication n'est pas totalement satisfaisante car elle ne rend compte ni de l'ampleur des changements ni de leur importance réelle. L'acquisition de nouveaux objets s'accompagne en effet d'un dépôt dans les tombes et cela en contradiction totale avec les usages de la période précédente. Dès lors, plutôt que de voir dans ces objets de simples éléments d'échange, je reste convaincu que nous avóns là le témoignage d'importantes modifications de comportement. L'installa-

30 Tombe 177 de Mailhac et tombe 149 d'Agde.

31 Voir à ce sujet les remarques de $P$. Lévêque in : Modes de contacts ..., op. cit., p. 427-428. 
tion d'un climat d'insécurité et la perception d'une menace conduisent les hommes à se faire désormais incinérer avec la panoplie des guerriers. Compte tenu du fait que les armes semblent, pour la plupart, fournies par les Grecs ou les Étrusques, ce n'est sans doute pas de ce côté-là qu'il faut chercher l'origine de la menace mais, peut-être bien à l'intérieur même du monde indigène. Dans cette perspective, le rôle qu'ont pu jouer les nouveaux arrivants se trouve modifié : sans être réellement passifs - ils fournissent des armes aux indigènes - ils sont, avant tout, les spectateurs de bouleversements qui touchent le monde indigène à la fin du viI ${ }^{e} \mathrm{~s}$., bouleversements dont ils seront, peut-être, à terme, les principaux bénéficiaires.

La nécropole de Pézenas permet également d'apporter de très importantes précisions sur une autre mutation qui marque en profondeur le monde indigène au début $\mathrm{du} \mathrm{vI}^{\mathrm{e}} \mathrm{s}$. : la modification des pratiques funéraires qui correspond au passage de la phase Ib à la phase II. Comme nous l'avons vu, cette dernière se caractérise par la forte réduction du mobilier funéraire, désormais composé de l'ossuaire accompagné d'un simple récipient à boire, coupe ou gobelet, et l'abandon total du dépôt de quartiers de viande non brûlés dans le fond des fosses. Cette mutation coïncide avec la généralisation de nouveaux objets métalliques, comme les boucles de ceinturon et les grands couteaux à dos arqué. On rappellera également qu'elle s'accompagne d'une modification dans le développement topographique de la nécropole avec la création d'un nouveau secteur d'ensevelissement (zone IIb), distinct du noyau primitif, au nord-est de la zone Ib.

Ces divers changements, que l'on observe tant dans le mobilier que dans les rites funéraires, sont évidemment le reflet de mouvements profonds qui affectent l'ensemble de notre groupe de population. Le grand intérêt de la nécropole de Pézenas est de les dater avec précision, les phases IIa et IIb devant être globalement situées dans la période 590-570. L'existence de quelques rares tombes de transition, situées à proximité immédiate de la zone $\mathrm{Ib}$, révèlent en outre que ces mutations sont très rapides et interviennent dès les débuts de cette période, très vraisemblablement aux environs de $590^{32}$. Ces chan-

32 La tombe 98 de la nécropole de Pézenas, datable des environs de 580, marque le terme de cette évolution. Après cette date, aucune tombe ne présentera à la fois le mobilier abondant et l'offrande alimentaire qui caractérisent les nécropoles de la période la plus ancienne de l'Age du F'er. gements ne sont d'ailleurs pas limités à la seule nécropole de Pézenas; ils ont pu être observés sur d'autres sites, en particulier à Mailhac, où ils correspondent au passage de la nécropole du Grand Bassin I à celle du Grand Bassin II. Il s'agit donc de bouleversements importants qui affectent sans doute l'ensemble des sociétés indigènes du Languedoc occidental. Rien ne nous permet, pour le moment, d'en connaître les causes ou l'ampleur réelle. S'agit-il d'une simple évolution des mentalités? Comment expliquer, dans ce cas, la rapidité des changements observés et l'importance même des zones géographiques concernées? S'agit-il, comme cela est plus vraisemblable, de mouvements plus profonds liés à un renouvellement partiel $\mathrm{du}$ fonds de population primitif? Dans ce cas, doit-on y voir les effets d'infiltrations progressives ou la conséquence de mouvements plus amples, voire plus brutaux? La multiplication des fouilles de nécropoles permettra peut-être, dans l'avenir, de répondre à ces questions. On peut cependant d'ores et déjà observer que c'est à partir de cette période que les rapprochements que l'on peut effectuer avec l'Espagne sont plus nombreux et plus significatifs. Les objets métalliques adoptés durant notre période IIa/IIb - boucles de ceinturon, grands couteaux - se retrouvent en effet en Espagne et témoignent au moins d'une parenté de civilisation plus accentuée que précédemment. Là encore, les questions surgissent: simple effet de contacts commerciaux accrus ou conséquence de déplacements, même limités, de populations? Force est ici d'avouer notre embarras car, même si l'on accepte cette dernière hypothèse, l'absence d'une chronologie précise des nécropoles du nord de l'Espagne ne permet pas de dire dans quel sens s'est effectué le mouvement. Le fait que la région d'Agde se trouve à la périphérie de l'aire de diffusion de ce type de mobilier montre cependant que ce n'est sans doute pas là qu'il convient de chercher le foyer d'origine de ces phénomènes, mais plutôt dans des zones plus méridionales, carrefour narbonnais ou nord de l'Espagne.

Rappelons enfin que c'est durant cette période IIa/IIb que l'on observe la première rupture, dans le développement topographique de cette nécropole, avec l'aménagement d'un secteur réservé où se concentrent les tombes de guerriers. Compte tenu du fait qu'aucune observation analogue n'a pu être faite, ni dans les parties plus anciennes ni dans les zones de développement plus récentes, il est permis de se demander si les changements que nous évoquions précédemment sont bien le fait d'infiltrations progressives ou pacifiques ou si, au contraire, 
l'aménagement d'un secteur réservé pour des tombes de guerriers ne doit pas être considéré comme le témoignage de bouleversements plus importants.

Image complexe, donc, que celle révélée par la nécropole de Pézenas pour ces débuts du vi's., période sans doute trouble, durant laquelle les populations de l'arrière-pays d'Agde subissent les contrecoups de deux mouvements d'origines diverses, internes au monde indigène pour une part, mais aussi externes, avec les effets d'une pression accrue exercée par des nouveaux arrivants, Grecs ou Étrusques.

Cette pression, sans doute relativement légère au tout début du $\mathbf{v I}^{\text {es. }}$., va aller en s'accentuant durant le deuxième quart du $\mathrm{vI}^{\mathrm{c}} \mathrm{s}$. avec la diffusion massive des premières productions de céramiques grises languedociennes. A la différence des vases à pâte claire et des canthares en bucchero, qui attestent simplement la permanence des contacts avec les navigateurs étrangers qui diffusent ce type de mobilier sur toute la bordure occidentale du golfe du I,ion, l'apparition des imitations de vases indigènes en céramique grise comporte une tout autre signification. Elle témoigne, en effet, de la création, sur les côtes mêmes du Languedoc occidental, d'ateliers de potiers grecs qui fabriquent el diffusent ce type de mobilier. La présence de ces productions dans la nécropole de Pézenas revêt donc un double intérêt: elle permet, en effet, non seulement de dater le développement de ces ateliers grecs locaux - les premiers cuvrent dès avant 580 - mais montre également que, dès le départ, les potiers grecs se sont adaptés au marché local, en fournissant aux indigènes ces cratères à pied haut qui, dans leur version non tournée, apparaissaient en si grande quantité dans les phases les plus anciennes de cette nécropole. Comment ne pas voir là les premiers signes de la modification des rapports entre les divers groupes humains? A une période d'exploration et d'observation, succèdent tout naturellement les premières tentatives d'exploitation, avec production de biens spécifiques, destinés à une clientèle indigène dont le retard technologique offre un débouché important à des céramiques de qualité pourtant médiocre. La nature même de ces productions, dont la technique - mais non la forme - est dérivée du bucchero gris de Grèce de l'Est, trahit d'ailleurs la part prise désormais par l'élément purement phocéen dans la fréquentation de cette région. Si pour les périodes anciennes la question reste ouverte - Grecs ou Etrusques, ou les deux -, à partir des années 580, il est clair que l'on assiste à une tentative de mainmise phocéenne. Parallèlement à l'adoption de ces premiè- res imitations en céramique grise, on note d'ailleurs une évolution très sensible du répertoire des formes indigènes, marquée notamment par la disparition quasi totale des cratères indigènes à pied haut. On aurait tort de ne voir là qu'une simple évolution du goût de la clientèle indigène. L'adoption des imitations grecques implique en effet l'instauration de rapports économiques d'un type nouveau, de liens de dépendance qui provoqueront la décadence rapide et durable de l'artisanat local. Un tel bouleversement des circuits économiques a pu, bien sûr, s'accompagner de profondes modifications des rapports sociaux dans notre groupe de population, modifications dont nous ne pouvons malheureusement, pour le moment, mesurer les effets réels.

Comme nous l'avons vu, ce n'est que vers 570 que les amphores étrusques apparaissent massivement dans la nécropole de Saint-Julien. L'utilisation tardive de ce type de récipient comme ossuaire ne doit pas donner lieu à des interprétations hâtives. Elle ne correspond nullement à un regain du commerce étrusque - dans ces régions la diffusion de ces produits peut très bien avoir été assurée par les Phocéens - et ne signifie pas non plus que ces récipients étaient inconnus avant cette date. Elle peut, en effet, s'expliquer tout simplement par le fait que ces récipients grossiers - en fait de simples conteneurs - ont longtemps été jugés indignes de figurer dans les tombes où traditionnellement on dépose des vases de prix, comme le montrent celles du début $d u v^{\mathrm{e}} \mathrm{s}$. Cette utilisation massive des amphores témoigne cependant incontestablement de la part importante prise par le commerce du vin dans les échanges avec les populations indigènes durant le deuxième quart du $\mathbf{v i}^{e} \mathbf{s}$. Ce point n'a pas été, à mon sens, assez souligné. Quand on connaît les ferments de dissolution sociale qu'introduit la consommation abondante d'alcool dans les sociétés primitives, il n'est pas absurde de penser que la fourniture de telles boissons ne procède pas seulement, de la part des nouveaux arrivants, de seules considérations économiques. La déstabilisation sociale et la dépendance qu'elle entraîne pouvaient, en effet, favoriser grandement la mainmise des commerçants étrangers sur cette région. La nécropole de Pézenas, dans le deuxième quart $\mathrm{du} \mathrm{VI}^{\mathrm{e}} \mathrm{s}$, , nous renvoie en fait l'image d'un groupe humain largement ouvert sur le monde méditerranéen, mais désormais intégré à un système d'exploitation de l'arrière-pays soigneusement mis en place par les commerçants phocéens.

Vers le milieu du $\mathrm{vI}^{\mathrm{e}}$ s., la nécropole se développe par rapport à un chemin central, dont on ne peut cependant dire s'il s'agit d'une simple desserte de l'aire funéraire ou d'une voie préexistan- 
te qui a été respectée par la nécropole. Au niveau même des sépultures, les tendances observées précédemment se renforcent encore. Le mobilier non tourné est désormais extrêmement rare; le répertoire typologique s'est considérablement appauvri, la production locale se cantonnant dans des formes simples et fonctionnelles. Le façonnage souvent hâtif ou maladroit est le produit d'un artisanat qui ne peut plus résister à l'invasion des céramiques tournées qui sont désormais extrêmement variées. La part prépondérante revient, là encore, aux céramiques grises d'origine régionale. La proportion des imitations de formes indigènes tend d'ailleurs à diminuer au profit de formes de type grec, œnochoés, coupes de type B2, urnes à fond annulaire. La clientèle indigène semble progressivement abandonner les formes ancestrales - sauf les cratères à pied haut - au profit des seules formes nouvelles utilisées par les commerçants grecs. C'est également durant cette période, vers le milieu du $\mathrm{vr}^{\mathrm{e}} \mathrm{s}$. que des tombes s'installent au milieu des zones d'ensevelissement les plus anciennes, Ia et $\mathrm{Ib}$, en bouleversant les sépultures primitives. Ces deux faits - adoption progressive des formes grecques et non respect des zones d'ensevelissement traditionnelles - sont évidemment très intéressantes et doivent être considérées comme les preuves d'une certaine perte d'identité culturelle.

Les renseignements dont nous disposons sur la dernière phase d'utilisation de la nécropole sont peu nombreux et d'interprétation difficile, compte tenu du très mauvais état de conservation des sépultures qui nous sont parvenues. Les grandes tendances observées précédemment semblent cependant se confirmer avec une part toujours prépondérante des céramiques grises issues d'ateliers locaux et une régression continue du mobilier non tourné. C'est durant cette ultime phase d'utilisation de la nécropole que l'on assiste à une nouvelle modification des rites funéraires avec la disparition complète de l'ossuaire. Le mobilier est désormais totalement brûlé sur le bûçher et les résidus de la crémation vases brisés, objets en métal, ossements calcinés sont déversés pêle-mêle dans de grandes fosses. En raison du mauvais état de conservation des secteurs concernés, la nécropole de Pézenas n'apporte aucun élément nouveau quant à l'agencement des tombes de cette période, mais confirme que cette pratique se généralise très rapidement vers 500 avant $J$.-C.

La nécropole de Saint-Julien est définitivement abandonnée dans les toutes premières années $d u \mathrm{v}^{*} \mathrm{~s}$. Seule une exploration approfondie de l'oppidum de Saint-Siméon permettrait, peut-être, de comprendre la signification de cet abandon qui, même s'il ne correspond pas à un abandon total ou partiel de l'habitat, témoigne au moins d'une rupture assez nette avec des pratiques et des usages ancestraux. Il sera d'ailleurs intéressant, à l'avenir, de voir si cette observation peut être mise en rapport avec un contexte plus général d'abandon ou de réoccupation d'habitats dans les premières années du v"s., qui lui conférerait, dès lors, une toute autre signification.

André Nickels 